\title{
SYMBOLIC MODELS FOR CONTROL SYSTEMS
}

\author{
PAULO TABUADA
}

\begin{abstract}
In this paper we provide a bridge between the infinite state models used in control theory to describe the evolution of continuous physical processes and the finite state models used in computer science to describe software. We identify classes of control systems for which it is possible to construct equivalent (bisimilar) finite state models. These constructions are based on finite, but otherwise arbitrary, partitions of the set of inputs or outputs of a control system.

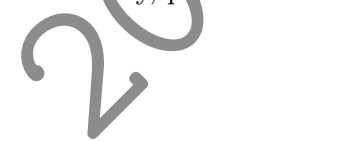

\section{INTRODUCTION}

1.1. Motivation. The formal specification, design and analysis of software systems is usually based on the use of finite state models such as transition systems, Buchi automata, etc. This fact creates a real challenge for embedded systems in which continuous components described by infinite state models, such as differential equations, interact with software modules. Although much is known about these hybrid systems, as can be seen from the proceedings of the latest workshops on the subject [HSC02, HSC03, HSC04, HSC05, HSC06], systematic design methodologies are still lacking. An this paper we propose an important step towards a "correct by design" synthesis theory for embedded systems by replacing infinite state models of physical systems with equivalent finite state models. The possibility of expressing the dynamics of control systems through finite state models offers great promises for new design techniques addressing the requirements of both continuous and discrete components. Not only these finite state models offer a common framework bridging control theory and computer science but they also enable the use of algorithmic synthesis techniques that are traditionally not considered in control theory.

1.2. Contributions. The existence of finite state models is addressed through two different perspectives: an input and an output perspective. The input perspective is based on the specification of a desired input set as a finite partition of the existing infinite input set. Each element of this partition can be identified with a symbol and the objective is to construct an equivalent control system having these symbols as inputs. This symbolic view of inputs is natural since embedded software/hardware can only influence the dynamics of the physical world through a finite set of inputs. We show that these control systems with symbolic inputs always exist and that under suitable assumptions (linearity and controllability for example) they have a finite number of states. The output perspective is identical to the input perspective except that the starting point is a finite partition of the infinite output set of a control system. This is also a natural starting point since embedded software/hardware can only react to the physical environment through a finite description of its state. Starting from such symbolic description of the output of a control system the objective is to construct the simplest equivalent control system generating the same symbolic outputs. Under the relevant technical assumptions (linearity and controllability for example) we show existence and finiteness of these models. These finiteness results encompass existing results by the author and coworkers [TP03b, TP03a, TP06] and also provide new examples of systems which were previously not known to admit symbolic models.

1.3. Related work. The idea of using symbolic models for the control of continuous systems is not new and motivated much research in the area of hybrid systems [NK93, ASL93, Lun94, SKHP97, CW98, RO98, VSS ${ }^{+}$01, MRO02, DMN02, KA03, TMD04]. Even though the use of symbolic models was advocated by these and many

This research was partially supported by the National Science Foundation CAREER award 0446716. 
other researchers, the applicability of the proposed methods has always remained an open problem due to the lack of results ensuring existence of symbolic models for control systems. Initial attempts to the construction of symbolic models for control systems were based on integrals of motion [SKA01, Bro99]. Integrals or constants of motion are a quite natural way of defining state space partitions compatible with the continuous dynamics. The results in this paper, however, apply to classes of systems for which constants of motion do not necessarily exist and can therefore be seen as complementary to existing work, especially to [Bro99]. Another line of inquiry also related to the work presented in this paper is the study of quantized control systems [BMP02, PLPB02]. Symbolic models based on input quantization represent a different approach to symbolic control. Quantized input models are obtained by selecting a countable subset of the input space contrasting with finite bisimulations which are obtained by selecting a finite partition of the state or input and state space. Even though this observation seems to suggest a certain duality between these approaches, further research is still required to attest and formalize such duality. A different but related line of research consists on the study of bisimulations of purely continuous [vdS04, Pap03, TP04] or hybrid systems [PvdSdB04, HTP05]. The work described in these references represents another approach to complexity reduction through the use of bisimulation based abstractions.

In the case of dynamical systems the situation is much better understood. Starting with Alur and Dill's work on timed automata [AD94], a fair amount of work was done to push the boundaries of the class of systems admitting finite bisimulations. This work culminated with the papers [PV94, ACH ${ }^{+}$95, HKPV98, HM00] introducing the decidable classes of multi-rate and rectangular hybrid automata. On the purely continuous side, we mention the work of Lafferriere and co-workers [LPS00] which used o-minimality to ensure existence of symbolic models. See also [BMRT04] for a simpler and more insightful proof of the same results. Unfortunately the techniques based on o-minimality do not extend to control systems since the space of inputs is usually infinite dimensional (for example the space of piece-wise constant functions) and thus fails to be captured by o-minimal structures.

1.4. Organization. The results presented in this paper were developed in the setting of category theory [Lan71] and in a framework very similar to the one used in [WN94] to compare different models for concurrency. However, for the sake of readability, we limited the use of category theory to the notion of morphism, essential for the results to be presented, and to the notions of completion. In particular, all the proofs are explicit and involve no category theoretic concepts. The readers averse to abstract constructions are invited to use the concrete results presented in Corollaries 4.9 and 5.6 as motivation for the abstract perspective taken in this paper

The paper is organized as follows. The class of control systems considered in this paper and the corresponding morphisms are introduced in Section 2 while the notion of bisimulation is discussed in Section 3 . Input induced bisimulations are the topic of Section 4 where their existence is proved. Finite bisimulations induced by inputs are also discussed and conditions for their existence established. Section 5 focuses on output induced bisimulations. Existence conditions are provided in this section for infinite and finite output induced bisimulations. The paper finishes with some discussion of the presented results in Section 6. For completeness, some results on equivalence relations are collected in an Appendix at the end of the paper.

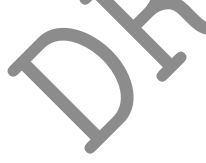

\section{Control Systems AND Their MORPhisms}

Control systems are usually defined by a differential equation:

$$
\frac{d}{d t} x=F(x, u)
$$

where $x \in \mathbb{R}^{n}$ denotes the state and $u \in \mathbb{R}^{m}$ denotes the input. Consider for example a block of mass $m$ attached to a wall through a spring as displayed in Figure 1. From basic physics we know that the sum of the forces acting on the block is given by $m a$ where $a$ is the acceleration of the block. The spring induces a 


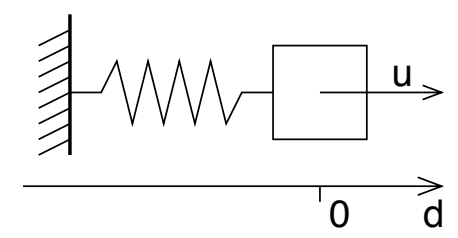

FiguRE 1. Block attached to a wall through a spring.

force $-K d$ proportional to the displacement $d$ of the block and where $K$ models the physical properties of the spring. If $u$ is an external force also acting on the block we have:

$$
m a=-K d+u
$$

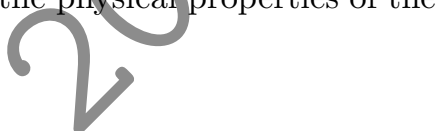

Noting that acceleration is the time derivative of velocity $v$ and that velocity is the time derivative of the displacement $d$ we arrive at the following differential equation describing the evolution of the block:

$$
\begin{aligned}
& \frac{d}{d t} v=-K d+\mu \\
& \frac{d}{d t} d=v
\end{aligned}
$$

We can thus regard this systems as a control system with $F$ and state $x$ given by:

and where $u \in \mathbb{R}$ is the input.

$$
F(x, u)=\left[\begin{array}{c}
-K x+u \\
v
\end{array}\right],\left[\begin{array}{l}
v \\
d
\end{array}\right] \in \mathbb{R}^{2}
$$

Under standard regularity conditions (see for example [Son98]) on the map $F: \mathbb{R}^{n} \times \mathbb{R}^{m} \rightarrow \mathbb{R}^{n}$ and on a curve $\mathbf{u}:[0, \tau] \rightarrow \mathbb{R}^{m}$ with $\tau>0$, there exists a curve $\mathbf{x}:[0, \tau] \rightarrow \mathbb{R}^{n}$ satisfying:

$$
\frac{d}{d t} \mathbf{x}(t)=F(\mathbf{x}(t), \mathbf{u}(t))
$$

for almost all $t \in[0, \tau]$. The curve $\mathbf{u}$ is considered an input trajectory and it is chosen in order to ensure that the resulting state trajectory $\mathbf{x}$ satisfies certain desirable properties. We may be interested, for example, in choosing $\mathbf{u}$ in order to guarantee that the block in Figure 1 moves from $d=0$ to some other desired displacement. If we denote the set of all such input trajectories by $U$ and if we denote by $f$ the map sending the pair $(\mathbf{x}(0), \mathbf{u})$ to $\mathbf{x}(\tau)$ we can regard a control system simply as a map:

$$
f: M \times U \rightarrow M
$$

where $M=\mathbb{R}^{n}$ is the state set and $U$ the input set. When $U$ only contains input trajectories defined on intervals of fixed length $\tau>0$, we shall regard $f: M \times U \rightarrow M$ as a discrete-time control system since, starting at time 0 , the map $f$ only describes the evolution of the continuous system for the discrete-time instants $0, \tau, 2 \tau, 3 \tau, \ldots$. This is the most common situation in applications where the input trajectory is the outcome of a periodically executed task with period $\tau$. When $U$ contains trajectories defined on intervals of all lengths $\tau \in \mathbb{R}$, then we regard $f: M \times U \rightarrow M$ as a continuous-time control system as it describes the evolution of the continuous system for every $\tau \in \mathbb{R}$. We shall not be too concerned with the distinction between discrete-time and continuous-time when developing our results. We note, however, that independently on time being treated as discrete or continuous, the set of states remains infinite ( $\mathbb{R}^{2}$ in the block example). Given the above discussion we shall model control systems as a map:

$$
f: M \times U \rightarrow M
$$


describing the state $f(x, u) \in M$ resulting from applying input $u \in U$ at the state $x \in M$. In many situations the resulting state $f(x, u)$ cannot be observed directly but rather through an output map:

$$
r: M \rightarrow P
$$

transforming states $x \in M$ into outputs $r(x) \in P$. This is the case in our block example if we only have a sensor measuring the displacement of the block. Although we regard the state as an element $(v, d) \in \mathbb{R}^{2}$, we only have access to sensor measurements providing the displacement $r(v, d)=d \in \mathbb{R}$.

We will usually denote a control system by the pair $(f, r)$ or by $M \times U \stackrel{f}{\longrightarrow} M \stackrel{r}{\longrightarrow} P$ when we want to emphasize the input, state and output sets. When $P=M$ and $r=1_{M}\left(1_{M}\right.$ is the identity function on $\left.M\right)$ we will simply denote a control system by $M \times U \stackrel{f}{\longrightarrow} M$. Since our ultimate objective is to obtain finite models for (2.1) we shall not require any additional structure on the sets $M, U$ and $P$ or on the maps $f$ and $r$.

Recall that in [WN94] a transition system is defined as a quadruple $(S, i, L$, Tran $)$ where $S$ is a set of states, $i \in S$ is a distinguished state, $L$ is a set of labels and Tran $\subseteq S \times L \times S$ is a transition relation. A control system $M \times U \stackrel{f}{\longrightarrow} M$ also defines a transition relation given by the graph of $f$, that is, $\Gamma(f)=\{(x, u, y) \in$ $M \times U \times M \mid f(x, u)=y\} \subset M \times U \times M$. We thus conclude that both models are very close in the way they capture the dynamics. However, control systems are not equipped with a distinguished initial state but they are equipped with an observation map $r: M \rightarrow P$. This distinguishes the models with respect to observations. For control systems, observations are obtained through the map $r$ acting on the state while for transition systems observations are directly available as the labels $l \in L$ of transitions in Tran.

The notion of morphism between control systems will be instrumental for the results developed in this paper. Intuitively, a morphism between control systems is a triple of maps describing how inputs, states and outputs of one system are related to inputs, states and outputs of another system.

Definition 2.1. Let $M \times U \stackrel{f}{\longrightarrow} M \stackrel{r}{\longrightarrow} P$ and $N \times V \stackrel{g}{\longrightarrow} N \stackrel{s}{\longrightarrow} Q$ be control systems. A morphism $\alpha:(f, r) \rightarrow(g, s)$ from $(f, r)$ to $(g, s)$ is given by a triple of maps $\alpha=\left(\alpha_{1}, \alpha_{2}, \alpha_{3}\right): M \times U \times P \rightarrow N \times V \times Q$ with $\alpha_{1}: M \rightarrow N, \alpha_{2}: M \times U \rightarrow V$ and $\alpha_{3}: P \wedge Q$, making the following diagram commutative:

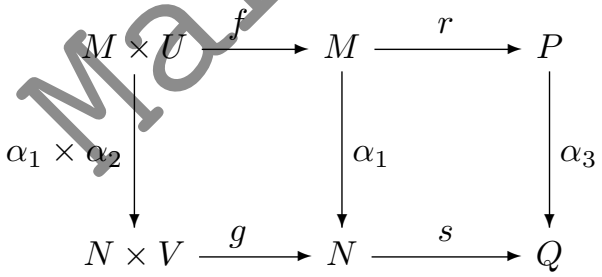

that is, satisfying the following equalities:

$$
\begin{aligned}
\alpha_{1} \circ f(x, u) & =g\left(\alpha_{1}(x), \alpha_{2}(x, u)\right) \\
\alpha_{3} \circ r & =s \circ \alpha_{1}
\end{aligned}
$$

When $P=M, Q=N, r=1_{M}$ and $s=1_{N}$ we shall denote a morphism $\alpha$ simply as a pair $\alpha=\left(\alpha_{1}, \alpha_{2}\right)$ : $M \times U \rightarrow N \times W$ ?

The introduced notion of morphism is also very close to the notion of morphism between transition systems in [WN94]. The important difference is that we work with totally defined maps and that the map $\alpha_{2}: M \times U \rightarrow$ $V$ mapping inputs (or labels) to inputs (or labels) is state dependent. It is easy to show that in both cases morphisms carry trajectories or traces of one system into trajectories or traces of another system.

In a morphism $\alpha=\left(\alpha_{1}, \alpha_{2}\right): f \rightarrow g$, map $\alpha_{2}$ can be many times determined from map $\alpha_{1}$ as the next result shows.

Proposition 2.2. Let $M \times U \stackrel{f}{\longrightarrow} M$ and $N \times V \stackrel{g}{\longrightarrow} N$ be control systems and let $\alpha_{1}: M \rightarrow N$ be a map. If the following inclusion holds:

$$
\alpha_{1} \circ f(x, U) \subseteq g\left(\alpha_{1}(x), V\right)
$$


there exists a map $\alpha_{2}: M \times U \rightarrow V$ such that $\left(\alpha_{1}, \alpha_{2}\right)$ is a morphism from $f$ to $g$. Furthermore, if $g_{y}: V \rightarrow N$ is injective for every $y \in \alpha_{1}(M)$, then $\alpha$ is unique.

Proof. Assume that $\alpha_{1} \circ f(x, U) \subseteq g\left(\alpha_{1}(x), V\right)$ holds. Then, for every $(x, u) \in M \times U, \alpha_{1} \circ f(x, u) \in g\left(\alpha_{1}(x), V\right)$ so that we can define $\alpha_{2}$ at $(x, u)$ to be any $v \in V$ satisfying:

$$
\alpha_{1} \circ f(x, u)=g\left(\alpha_{1}(x), v\right)
$$

This construction immediately provides a map $\alpha_{2}: M \times U \rightarrow V$ making $\left(\alpha_{1}, \alpha_{2}\right)$ a morphism from $f$ to $g$. Assume now that $g_{y}$ is injective for every $y \in \alpha_{1}(M)$. Then, the choice of $v \in V$ satisfying (2.6) is unique thus defining $\alpha_{2}$ uniquely.

We will also need to analyze the behavior of $f$ in several steps and the following notation will be very useful in that regard. We denote by $U^{*}$ the monoid freely generated by $U$, that is, the set of all finite length strings obtained by concatenating elements in $U$. Note that the string of zero length, denoted by $\varepsilon$, also belongs to $U^{*}$. We will also have the opportunity to use $U^{\leq k}$ to denote the set of all strings of length smaller or equal than $k \in \mathbb{N}$. If $u=u_{1} u_{2} \ldots u_{n} \in U^{*}$, we will use $u(i)$ to denote the $i$ th element of the string $u$, that is, $u(i)=u_{i}$ with the convention that $u(0)=\varepsilon$ and we will use $u[i]$ to denote the first $i$ elements of $u$, that is, $u[i]=u_{1} u_{2} \ldots u_{i}$. Several maps extend in a unique way to the sets $U^{*}$ and $U^{\leq k}$. Map $f^{*}: M \times U^{*} \rightarrow M$ is defined by $f^{*}(x, \varepsilon)=x$ and by the recursion $f^{*}(x, u)=f^{*}(f(x, u(1)), u(2) u(3) \ldots u(n))$ for any string $u$ of length $n$. Map $f^{\leq k}: M \times U^{\leq k} \rightarrow M$ is the restriction of $f^{*}$ to $M \times U^{\leq k}$. We will also use $\alpha_{2}^{*}: M \times U^{*} \rightarrow V^{*}$ to denote the extension of $\alpha_{2}: M \times U \rightarrow V$ defined by $\alpha_{2}^{*}(x, \varepsilon)=x$ and by the recursion $\alpha_{2}^{*}(x, u)=\alpha_{2}(x, u(1)) \alpha_{2}^{*}(f(x, u(1)), u(2) \ldots u(n))$ for any string $u$ of length $n$. The notation $f_{x}$ and $\alpha_{2 x}$ will be used to denote the functions defined by $f_{x}(u)=f(x, u)$ and $\alpha_{2 x}(u)=\alpha_{2}(x, u)$, respectively. Other notational conventions used through the paper are as follows. For a map $\phi: A \rightarrow B$ we will denote by $\phi(A), \phi^{-1}(b)$ and $\phi^{-1}(C)$ with $b \in B$ and $C \subseteq B$, the sets $\cup_{a \in A}\{\phi(a)\},\{a \in A \mid \phi(a)=b\}$ and $\cup_{c \in C} \phi^{-1}(c)$, respectively, and $|A|$ will denote the cardinality of set $A$.

\section{MORphisms And Bisimulations}

The notion of bisimulation [Par81, Mil89] is usually defined by resorting to transition systems as operational models. Although control systems also define transition relations we shall define bisimulation relations directly for control systems:

Definition 3.1. Let $M \times U \stackrel{f}{\longrightarrow} M \stackrel{r}{\longrightarrow} O$ and $N \times V \stackrel{g}{\longrightarrow} N \stackrel{s}{\longrightarrow} O$ be control systems with the same output set $O$. A relation $R \subseteq M \times N$ is said to be a bisimulation relation between $(f, r)$ and $(g, s)$ if for every $\left(x_{1}, y_{1}\right) \in R$ the following holds:

(1) $r\left(x_{1}\right)=s\left(y_{1}\right)$;

(2) for any $u \in U, f\left(x_{1}, u\right)=x_{2}$ implies the existence of $v \in V$ such that $g\left(y_{1}, v\right)=y_{2}$ and $\left(x_{2}, y_{2}\right) \in R$;

(3) for any $v \in V, g\left(y_{1}, v\right)=y_{2}$ implies the existence of $u \in U$ such that $f\left(x_{1}, u\right)=x_{2}$ and $\left(x_{2}, y_{2}\right) \in R$.

There is an important difference between this notion and the one introduced in [Par81, Mil89]. We require related states to match on outputs but we do not require transitions to match on labels. This modeling choice is motivated by control applications where the state carries important physical information about the system and the desired behavior is described in terms of the state evolution. The input, however, is a signal that we can arbitrarily manipulate in order to enforce the desired output behavior. It is not difficult to see that bisimilar control systems exhibit the same output behavior and that this notion of bisimulation is still a congruence with respect to parallel composition ${ }^{1}$.

\footnotetext{
${ }^{1}$ The relevant notion of parallel composition forces the intervening control systems to synchronize on outputs rather than on transition labels.
} 
Instead of working with Definition 3.1 it will be more convenient, at the technical level, to work with the following class of morphisms.

Definition 3.2. A morphism $\alpha:(f, r) \rightarrow(g, s)$ from control system $M \times U \stackrel{f}{\longrightarrow} M \stackrel{r}{\longrightarrow} P$ to control system $N \times V \stackrel{g}{\longrightarrow} N \stackrel{s}{\longrightarrow} Q$ is said to be fiber surjective if $\alpha_{2}: M \times U \rightarrow V$ is fiber surjective, that is, $\alpha_{2 x}: U \rightarrow V$ is surjective for every $x \in M$. Morphism $\alpha$ is said to be surjective if it is fiber surjective and both $\alpha_{1}: M \rightarrow N$ and $\alpha_{3}: P \rightarrow Q$ are surjective maps.

The choice of fiber-surjective morphisms is justified by the following result.

Proposition 3.3. Let $\alpha:(f, r) \rightarrow(g, s)$ be a fiber surjective morphism from control system $M \times U \stackrel{f}{\longrightarrow} M \stackrel{r}{\longrightarrow} P$ to control system $N \times V \stackrel{g}{\longrightarrow} N \stackrel{s}{\longrightarrow} Q$. The graph of the map $\alpha_{1}$, defined by $\Gamma\left(\alpha_{1}\right)=\{(x, y) \in$ $\left.M \times N \mid \alpha_{1}(x)=y\right\}$, is a bisimulation relation between control system $M \times U \rightarrow \stackrel{f}{\longrightarrow} M \stackrel{\alpha_{3} \text { or }}{\longrightarrow} Q$ and control system $N \times V \stackrel{g}{\longrightarrow} N \stackrel{s}{\longrightarrow} Q$.

Proof. Let $(x, y) \in \Gamma\left(\alpha_{1}\right)$. Then $\alpha_{1}(x)=y$ and it follows from (2.5) that $\alpha_{3} \circ r\left(x_{1}\right)=s \circ \alpha_{1}(x)=s(y)$ thus showing that requirement (1) in Definition 3.1 is satisfied.

Assume now that $f\left(x_{1}, u\right)=x_{2}$ and $\alpha_{1}\left(x_{1}\right)=y_{1}$. Then by $(2.5)$ we have $\alpha_{1}\left(x_{2}\right)=\alpha_{1} \circ f\left(x_{1}, u\right)=$ $g\left(\alpha_{1}\left(x_{1}\right), \alpha_{2}\left(x_{2}, u\right)\right)=g\left(y_{1}, v\right)$ thus showing that requirement $(2)$ in Definition 3.1 also holds with $v=$ $\alpha_{2}\left(x_{1}, u\right)$.

Finally we prove requirement (3). Let $\alpha_{1}\left(x_{1}\right)=y_{1}$ and $g\left(y_{1}, v\right)=y_{2}$. Since $\alpha$ is fiber surjective, there exists $u \in U$ satisfying $\alpha_{2}\left(x_{1}, u\right)=v$. If we denote by $x_{2}$ the state $f\left(x_{1}, u\right)$, it follows from (2.4) that $\alpha_{1}\left(x_{2}\right)=\alpha_{1} \circ f\left(x_{1}, u\right)=g\left(\alpha_{1}\left(x_{1}\right), \alpha_{2}\left(x_{1}, u\right)\right)=g\left(y_{1}, v\right)=y_{2}$ which concludes the proof.

Note that not every bisimulation can be obtained as the graph of a fiber-surjective morphism. However, this kind of bisimulation will suffice for the results presented in this paper.

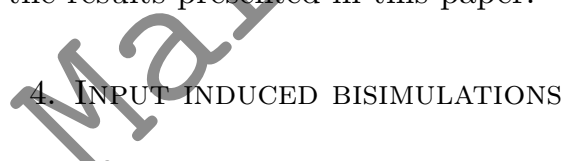

In this section we discuss the construction of bisimilar control systems induced by inputs. Consider the block example described in the previous section and assume we want to develop a controller, to be implemented in embedded hardware/software, whose objective is to force the block to a desired displacement different from zero. This controller will change the displacement of the block through an actuator exerting a force $u$ on the block. However, the actuator can only enforce ${ }^{2}$ a finite number of force values. Let us assume that these values are $-1,0$ and 1 and that the actuator transforms requests into forces according to the following map:

$$
\alpha_{2}(x, u)=\left\{\begin{aligned}
-1 & \text { if } u \leq-1 \\
0 & \text { if }-1<u<1 \\
1 & \text { if } u \geq 1
\end{aligned}\right.
$$

It is therefore natural to construct a bisimilar control system with input set $V=\{-1,0,1\}$ describing the dynamics of the original control system under these new inputs.

4.1. Existence of input induced bisimulations. Starting from a control system $M \times U \stackrel{f}{\longrightarrow} M \stackrel{r}{\longrightarrow} P$ and a map $\alpha_{2}: M \times U \rightarrow V$ there may exist several possible ways to obtain a control system $N \times V \stackrel{g}{\longrightarrow} N \stackrel{s}{\longrightarrow} Q$ and a morphism $\alpha:(f, r) \rightarrow(g, s)$. To make the problem well defined we shall require a unique factorization property.

\footnotetext{
${ }^{2}$ Alternatively we can assume that although the actuator can generate a force with an infinite number of intensities, it can only receive finitely many different requests from the software implementation of a controller.
} 
Definition 4.1. Let $M \times U \stackrel{f}{\longrightarrow} M \stackrel{r}{\longrightarrow} P$ be a control system and $\alpha_{2}: M \times U \rightarrow V$ a map. The $\alpha_{2^{-}}$ completion of $(f, r)$ is a triple $\left((g, s), \alpha_{1}, \alpha_{3}\right)$ where $N \times V \stackrel{g}{\longrightarrow} N \stackrel{s}{\longrightarrow} Q$ is a control system, $\alpha_{1}: M \rightarrow N$ and $\alpha_{3}: P \rightarrow Q$ are maps making $\alpha=\left(\alpha_{1}, \alpha_{2}, \alpha_{3}\right): M \times U \times P \rightarrow N \times V \times Q$ a morphism from $(f, r)$ to $(g, s)$ with the following property:

for any control system $N^{\prime} \times V \stackrel{g^{\prime}}{\longrightarrow} N^{\prime} \stackrel{s^{\prime}}{\longrightarrow} Q^{\prime}$ and morphism $\alpha^{\prime}=\left(\alpha_{1}^{\prime}, \alpha_{2}, \alpha_{3}^{\prime}\right):(f, r) \rightarrow\left(g^{\prime}, s^{\prime}\right)$ there exists a unique morphism $\beta=\left(\beta_{1}, 1_{V}, \beta_{3}\right):(g, s) \rightarrow\left(g^{\prime}, s^{\prime}\right)$ satisfying $\alpha^{\prime}=\beta \circ \alpha$.

Note that bisimilarity between $f$ and its $\alpha_{2}$-completion now follows trivially if one choses $\alpha_{2}$ to be fiber surjective. However, we shall study $\alpha_{2}$-completions for maps $\alpha_{2}$ that are not necessarily fiber-surjective since this extra generality adds no additional complications.

To understand the unique factorization property required by Definition 4.1 let us consider a control system $M \times U \stackrel{f}{\longrightarrow} M$ with $M=\left\{x_{1}, x_{2}, x_{3}\right\}, U=\left\{u_{1}, u_{2}, u_{3}\right\}$ and $f$ defined on Table 1 and also represented in Figure 2. If the map $\alpha_{2}: M \times U \rightarrow V$ is defined by $\alpha_{2}\left(x, u_{1}\right)=v_{1}=\alpha_{2}\left(x, u_{2}\right)$ and $\alpha_{2}\left(x, u_{3}\right)=v_{2}$ for every $x \in M$ and if $V=\left\{v_{1}, v_{2}\right\}$, then control systems $N \times V \stackrel{g}{\longrightarrow} N$ and $O \times V \stackrel{h}{\longrightarrow} O$ defined on Table 2 and represented in Figure 3 can be equipped with morphisms $\alpha=\left(\alpha_{1}, \alpha_{2}\right): f \rightarrow g$ and $\gamma=\left(\gamma_{1}, \alpha_{2}\right): f \rightarrow h$ also defined on Table 2 .

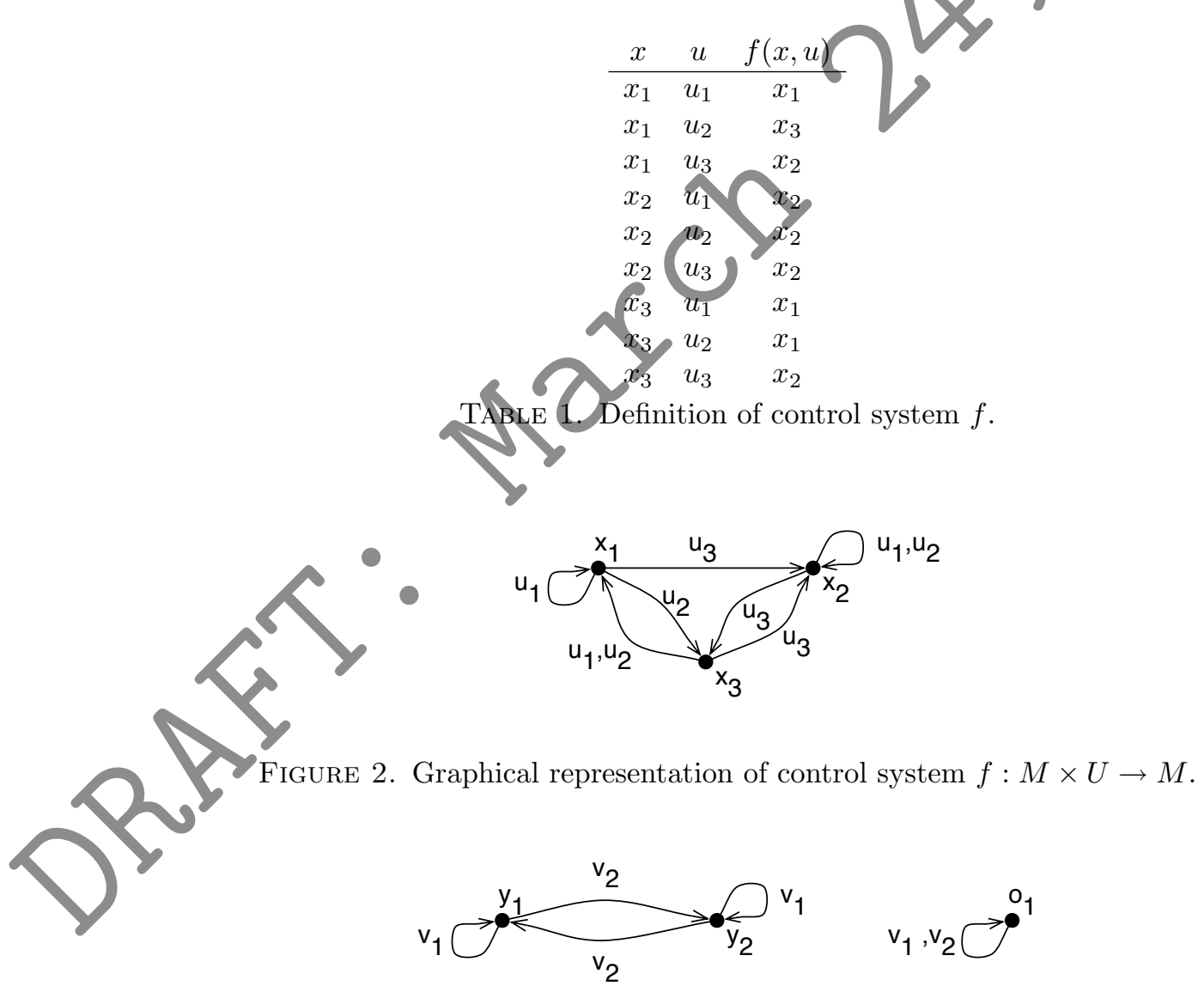

Figure 3. Graphical representation of control systems $g: N \times V \rightarrow N$ and $h: O \times V \rightarrow O$.

Among all the possible control systems equipped with a morphism from $f$, of which $N \times V \stackrel{g}{\longrightarrow} N$ and $O \times$ $V \stackrel{h}{\longrightarrow} O$ are two examples, the unique factorization property in Definition 4.1 isolates the "least constrained" 


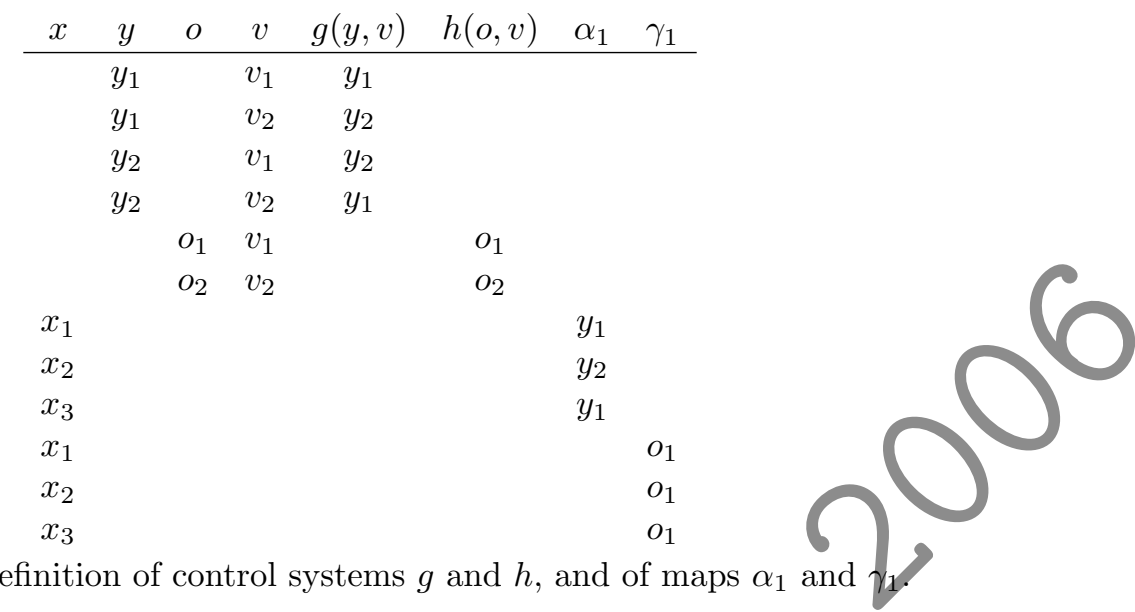

TABle 2. Definition of control systems $g$ and $h$, and of maps $\alpha_{1}$ and

solution. To illustrate this point, let us assume that $O \times V \stackrel{h}{\longrightarrow} O$ satisfies the unique factorization property. Then, there must exist a unique morphism $\beta=\left(\beta_{1}, 1_{V}\right): h \rightarrow g$ satisfying $\beta \circ \gamma=\alpha$. However, no such morphism exists since if $\beta_{1}\left(o_{1}\right)=y_{1}$, then $\beta_{1} \circ h\left(o_{1}, v_{2}\right)=\beta_{1}\left(o_{1}\right)=y_{1} \neq y_{2}=g\left(\beta_{1}\left(o_{1}\right), v_{2}\right)$. Similarly, if $\beta_{1}\left(o_{1}\right)=y_{2}$ we have $\beta_{1} \circ h\left(o_{1}, v_{2}\right)=\beta_{1}\left(o_{1}\right)=y_{1} \neq y_{2}=g\left(\beta_{1}\left(o_{1}\right), v_{2}\right)$. On the other hand it is not difficult to see that there exists a unique morphism $\beta$ from $g$ to $h$ given by $\beta_{1}\left(y_{1}\right)=o_{1}=\beta_{1}\left(y_{2}\right)$. Control system $h$ is thus over-constrained in the sense that too many states are mapped by the map $\gamma_{1}$ into the same state. It is in this sense that we can see the unique factorization requirement in Definition 4.1 as the specification of the least constrained control system equipped with a morphism from $(f, r)$.

The following result shows that output maps have no contribution for the existence of $\alpha_{2}$-completions. We shall therefore assume throughout this section that output sets equal the state sets and that output maps are the identity.

Proposition 4.2. Consider control system $M \times U \stackrel{f}{\longrightarrow} M \stackrel{1_{M}}{\longrightarrow} M$ and the map $\alpha_{2}: M \times U \rightarrow V$. If the $\alpha_{2}$-completion of $\left(f, 1_{M}\right)$ exists, then the $\alpha_{2}$-completion of $M \times U \stackrel{f}{\longrightarrow} M \stackrel{r}{\longrightarrow} P$ also exists for any output map $r: M \rightarrow P$.

Proof. Let $N \times V \stackrel{g}{\longrightarrow} \stackrel{\Im}{\longrightarrow} Q$ be the $\alpha_{2}$-completion of $\left(f, 1_{M}\right)$. We shall construct the $\alpha_{2}$-completion of $(f, r)$ from $g$. Consider the maps $r: M \rightarrow P$ and $\alpha_{1}: M \rightarrow N$. There is a unique ${ }^{3}$, up to isomorphism, way of constructing $\alpha_{3}: P \rightarrow R$ and $t: N \rightarrow R$ satisfying $\alpha_{3} \circ r=t \circ \alpha_{1}$. Let $X=P+N$ (+ denotes disjoint union) and let $S$ be the equivalence relation on $X$ generated by the pairs $\left(\alpha_{1}(x), r(x)\right)$ for every $x \in M$. Define $R=X / S, \alpha_{3}=\pi_{S} \circ i_{P}$ and $t=\pi_{R} \circ i_{N}$ where $i_{P}: P \rightarrow X$ and $i_{N}: N \rightarrow X$ are the natural inclusions and $\pi_{S}: X \rightarrow R=X / S$ is the natural projection. The equality $\alpha_{3} \circ r=t \circ \alpha_{1}$ follows immediately by construction of $R, \alpha_{3}$ and $t$. Furthermore, maps $\alpha_{3}$ and $t$ have the following property. For any other maps $a: P \rightarrow T$ and $b: N \rightarrow T$ there exists one and only one map:

$$
c: R \rightarrow T
$$

satisfying:

$$
\begin{aligned}
a & =c \circ \alpha_{3} \\
b & =c \circ t
\end{aligned}
$$

We leave to the reader to verify that the desired unique map $c$ is given by $c([p])=a(p)$ and $c([n])=b(n)$ for any $p \in P$ and $n \in N$.

\footnotetext{
${ }^{3}$ What we are about to construct is the push-out of $r: M \rightarrow P$ and $\alpha_{1}: M \rightarrow N$.
} 
We now claim that $\left((g, t), \alpha_{1}, \alpha_{3}\right)$ is the $\alpha_{2}$-completion of $(f, r)$. It is obvious that $\alpha$ is a morphism from $(f, r)$ to $(g, t)$ since $\left(\alpha_{1}, \alpha_{2}\right)$ satisfy (2.4) and $\alpha_{3}$ satisfies (2.5). Let now $\left(g^{\prime}, s^{\prime}\right)$ be any other control system equipped with a morphism $\left(\alpha_{1}^{\prime}, \alpha_{2}, \alpha_{3}^{\prime}\right):(f, r) \rightarrow\left(g^{\prime}, s^{\prime}\right)$. Since $(g, s)$ is the $\alpha_{2}$-completion of $(f, r)$ there exists one and only one map $\beta_{1}: N \rightarrow N^{\prime}$ making $\left(\beta_{1}, 1_{V}\right)$ a morphism from $g$ to $g^{\prime}$ and satisfying $\left(\beta_{1}, 1_{V}\right) \circ\left(\alpha_{1}, \alpha_{2}\right)=\left(\alpha_{1}^{\prime}, \alpha_{2}\right)$. To conclude the proof we only need to show existence and uniqueness of $\beta_{3}: R \rightarrow P^{\prime}$ satisfying $\beta_{3} \circ t=s^{\prime} \circ \beta_{1}$ and $\beta_{3} \circ \alpha_{3}=\alpha_{3}^{\prime}$. But this follows at once by taking $a=\alpha_{3}^{\prime}, b=s^{\prime} \circ \beta_{1}$ in (4.3) and (4.4), and $\beta_{3}=c$.

We now arrive at one of the main contributions of this section.

Theorem 4.3. The $\alpha_{2}$-completion of a control system $M \times U \stackrel{f}{\longrightarrow} M$ exists for any map $\alpha_{2}: M \times U \rightarrow V$.

Theorem 4.3 shows that bisimilar control systems induced by $\alpha_{2}$ maps always exist in the class of control systems considered in this paper. Furthermore, when $\alpha_{2}$ is fiber-surjective, the resulting $\alpha_{2}$-completion is guaranteed to be bisimilar to $(f, r)$.

Proof. The proof of Theorem 4.3 makes essential use of the following lemma which essentially states that the forgetful functor mapping control systems $M \times U \stackrel{f}{\longrightarrow} M$ to the state set $M$ and morphisms $\left(\alpha_{1}, \alpha_{2}\right)$ : $M \times U \rightarrow N \times U$ to $\alpha_{1}: M \rightarrow N$ has a left-adjoint on the subcategory of control systems with a fixed input set $U$. The existence of such adjoint underlies many of the generalizations of realization theory to more abstract contexts [Gog73, AM74].

Lemma 4.4. Let $\varkappa:\left(M \times V^{*}\right) \times V \rightarrow\left(M \times V^{*}\right)$ be the control system defined by $\varkappa\left(\left(x, v_{1}\right), v_{2}\right)=\left(x, v_{1} v_{2}\right)$. For any map $\gamma: M \rightarrow N$ and for any control system $N \times V \stackrel{g}{\longrightarrow} N$ there exists a unique morphism $\delta=\left(\delta_{1}, 1_{V}\right): \varkappa \rightarrow g$ such that $\delta_{1}(x, \varepsilon)=\gamma(x)$.

Proof of Lemma 4.4. Map $\delta_{1}$ is defined by $\delta_{1}(x, v)=g^{*}(\gamma(x), v)$. This choice results in a morphism since:

$$
\begin{aligned}
\delta_{1} \circ \varkappa\left(\left(x, v_{1}\right), v_{2}\right)=\delta_{1}\left(x, v_{1} v_{2}\right) & \text { by definition of } \varkappa \\
=g^{*}\left(\gamma(x), v_{1} v_{2}\right) & \text { by definition of } \delta_{1} \\
=g\left(g^{*}\left(\gamma(x), v_{1}\right), v_{2}\right) & \text { by definition of } g^{*} \\
=g\left(\delta_{1}\left(x, v_{1}\right), 1_{V}\left(v_{2}\right)\right) & \text { by definition of } \delta_{1}
\end{aligned}
$$

Furthermore $\delta_{1}(x, \varepsilon)=g^{*}(\gamma(x), \varepsilon)=\gamma(x)$. To show uniqueness consider any other morphism $\left(\delta_{1}^{\prime}, 1_{V}\right): \varkappa \rightarrow g$. We immediately have $\delta_{1}^{\prime}(x, \varepsilon)=\gamma(x)=\delta_{1}(x, \varepsilon)$. For any $(x, v) \in M \times V^{*}$ equality between $\delta_{1}^{\prime}$ and $\delta_{1}$ follows from:

$\begin{aligned} \delta_{1}^{\prime}(x, v)=\delta_{1}^{\prime} \circ \varkappa((x, \varepsilon), v) & \text { by definition of } \varkappa \\ =g\left(\delta_{1}^{\prime}(x, \varepsilon), 1_{V}(v)\right) & \text { since }\left(\delta_{1}^{\prime}, 1_{V}\right) \text { is a morphism } \\ =g(\gamma(x), v) & \text { since } \delta_{1}^{\prime}(x, \varepsilon)=\gamma(x) \\ =g\left(\delta_{1}(x, \varepsilon), 1_{V}(v)\right) & \text { since } \delta_{1}(x, \varepsilon)=\gamma(x) \\ =\delta_{1} \circ \varkappa((x, \varepsilon), v) & \text { since }\left(\delta_{1}, 1_{V}\right) \text { is a morphism } \\ =\delta_{1}(x, v) & \text { by definition of } \varkappa\end{aligned}$

We now return to the proof of Theorem 4.3

The desired completion $N \times V \stackrel{g}{\longrightarrow} N$ will be obtained from control system $\varkappa$ defined in Lemma 4.4 . The state set $N$ is defined as the quotient of $M \times V^{*}$ by the equivalence relation $R$ generated by the pairs:

$$
\left((f(x, u), \varepsilon),\left(x, \alpha_{2}(x, u)\right)\right)
$$


and invariant under the family of maps maps $\left\{\varkappa_{v}\right\}_{v \in V}$ (invariant under $\varkappa$ for short). Map $\alpha_{1}$ is the composition $\pi_{R} \circ i$ where $i: M \rightarrow M \times V^{*}$ is the inclusion $i(x)=(x, \varepsilon)$. Finally, we define control system $g$ by:

$$
g\left(\left[\left(x, v_{1}\right)\right], v_{2}\right)=\left[\left(x, v_{1} v_{2}\right)\right]=\left[\varkappa\left(\left(x, v_{1}\right), v_{2}\right)\right]
$$

It follows from invariance of $R$ under $h$ that $g$ is well defined. To show that $\alpha$ is indeed a morphism consider any $(x, u) \in M \times U$. The following sequence of identities:

$$
\begin{aligned}
\alpha_{1} \circ f(x, u)=\pi_{R} \circ i \circ f(x, u) & \\
=\pi_{R}(f(x, u), \varepsilon) & \text { by definition of } i \\
=[(f(x, u), \varepsilon)] & \text { by definition of } \pi_{R} \\
=\left[\left(x, \alpha_{2}(x, u)\right)\right] & \text { by }(4.5) \\
=\left[\varkappa\left((x, \varepsilon), \alpha_{2}(x, u)\right)\right] & \text { by definition of } \varkappa \\
=g\left([(x, \varepsilon)], \alpha_{2}(x, u)\right) & \text { by definition of } g \\
=g\left(\pi_{R} \circ i(x), \alpha_{2}(x, u)\right) & \text { by definition of } i \text { and } \pi_{R} \\
=g\left(\alpha_{1}(x), \alpha_{2}(x, u)\right) & \text { by definition of } \alpha_{1}
\end{aligned}
$$

shows that $\alpha$ is a valid morphism.

Consider now any other control system $g^{\prime}: N^{\prime} \times V \rightarrow N^{\prime}$ and any other map $\alpha_{1}^{\prime} ; M \rightarrow N^{\prime}$ making $\alpha^{\prime}=\left(\alpha_{1}^{\prime}, \alpha_{2}\right)$ a morphism from $f$ to $g^{\prime}$. Map $\alpha_{1}^{\prime}$ factors as $\alpha_{1}^{\prime}=\delta_{1} \circ i$ where $i: M \rightarrow M \times V^{*}$ is the inclusion $i(x)=(x, \varepsilon)$ and $\delta_{1}$ the unique map $\delta_{1}: M \times V^{*} \rightarrow N^{\prime}$ whose existence is asserted by Lemma 4.4. Map $\alpha_{1}: M \rightarrow N$ also factors as $\alpha_{1}=\pi_{R} \circ i$. Provided that we can show that:

$$
\pi_{R}\left(x_{1}, v_{1}\right)=\pi_{R}\left(x_{2}, v_{2}\right) \quad \Rightarrow \quad \delta_{1}\left(x_{1}, v_{1}\right)=\delta_{1}\left(x_{2}, v_{2}\right)
$$

we can define $\beta: N \rightarrow N^{\prime}$ by $\beta([(x, v)])=\delta_{1}(x, v)$. This is what we will now show by proving that the equivalence relation $S$ on $M \times V^{*}$ defined by $\left(\left(x_{1}, v_{1}\right),\left(x_{2}, v_{2}\right)\right) \in S$ when $\delta_{1}\left(x_{1}, v_{1}\right)=\delta_{1}\left(x_{2}, v_{2}\right)$ is invariant under $\varkappa$ and contains the pairs:

$$
\left((f(x, u), \varepsilon),\left(x, \alpha_{2}(x, u)\right)\right)
$$

Invariance under $\varkappa$ follows immediately from the fact that $\left(\delta_{1}, 1_{V}\right)$ is a morphism from $\varkappa$ to $g^{\prime}$. Consider now an arbitrary point $(x, u) \in M \times U$. From the fact that $\alpha^{\prime}: f \rightarrow g^{\prime}$ is a morphism we have:

Since $\alpha^{\prime}$ factors as $\alpha^{\prime}=\left(\delta_{1}, 1_{V}\right) \circ\left(i, \alpha_{2}\right)$ :

$$
\alpha_{1}^{\prime} \circ f(x, u)=g^{\prime}\left(\alpha_{1}^{\prime}(x), \alpha_{2}(x, u)\right)
$$

$$
\delta_{1} \circ i \circ f(x, u)=g^{\prime}\left(\delta_{1} \circ i(x), \alpha_{2}(x, u)\right)
$$

We now use the fact that $\left(\delta_{1}, 1_{V}\right)$ is a morphism from $h$ to $g^{\prime}$ to conclude:

$$
\begin{array}{cl}
\delta_{1}(f(x, u), \varepsilon)=\delta_{1} \circ i \circ f(x, u) & \text { by definition of } i \\
=g^{\prime}\left(\delta_{1} \circ i(x), 1_{V} \circ \alpha_{2}(x, u)\right) & \text { since }\left(\delta_{1}, 1_{V}\right) \circ\left(i, \alpha_{2}\right)=\alpha^{\prime} \text { is a morphism from } f \text { to } g^{\prime} \\
=g^{\prime}\left(\delta_{1}(x, \varepsilon), \alpha_{2}(x, u)\right) & \text { by definition of } i \\
=\delta_{1} \circ \varkappa\left((x, \varepsilon), \alpha_{2}(x, u)\right) & \text { since }\left(\delta_{1}, 1_{V}\right) \text { is a morphism from } \varkappa \text { to } g^{\prime} \\
=\delta_{1}\left(x, \alpha_{2}(x, u)\right) & \text { by definition of } \varkappa
\end{array}
$$

We thus conelude that $S$ is invariant under $\varkappa$ and contains the pairs:

$$
\left((f(x, u), \varepsilon),\left(x, \alpha_{2}(x, u)\right)\right)
$$

Since $R$ is the coarsest equivalence relation satisfying these two properties it follows that (4.7) holds and $\beta_{1}: N \rightarrow N^{\prime}$ is well defined. The fact that $\left(\beta_{1}, 1_{V}\right)$ is a morphism and unique is inherited from the same properties of $\left(\delta_{1}, 1_{V}\right)$ proved in Lemma 4.4. The proof is therefore complete.

Throughout the remaining paper we will be especially interested in $\alpha_{2}$-completions for fiber surjective maps $\alpha_{2}$. In this case, $\alpha_{2}$-completions admit the following simplified description that will later be used to establish existence of symbolic completions. 
Proposition 4.5. The $\alpha_{2}$-completion of control system $M \times U \stackrel{f}{\longrightarrow} M$ for a fiber surjective map $\alpha_{2}$ : $M \times U \rightarrow V$ admits the following description:

(1) $N=M / S$ where $S$ is the coarsest equivalence relation on $M$ satisfying for any $x \in M$ :

$$
\alpha_{2}^{*}\left(x, u_{1}\right)=\alpha_{2}^{*}\left(x, u_{2}\right) \quad \Rightarrow \quad\left(f^{*}\left(x, u_{1}\right), f^{*}\left(x, u_{2}\right)\right) \in S
$$

(2) $\alpha_{1}=\pi_{S}: M \rightarrow M / S=N$;

(3) $g$ is defined by $g(y, v)=\alpha_{1} \circ f(x, u)$ for any $(x, u) \in M \times U$ such that $\alpha(x, u)=(y, v)$.

Proof. Consider the equivalence relation $R$ on $M \times V^{*}$ defined by (4.5) in the proof of Theorem 4.3. The proof will consist in showing that every $(x, v) \in M \times V^{*}$ is equivalent under $R$ to $\left(f^{*}(x, u)\right.$, \&) for any $u \in U$ such that $\alpha_{2}^{*}(x, u)=v$. This equivalence allows to identify each equivalence class of $R$ with an element of the form $\left(f^{*}(x, u), \varepsilon\right)$ thus showing that we can equivalently describe $R$ by equivalence relation $S$. The proof will be done by induction on the length of $v$.

For $|v|=1$ the result follows from (4.5).

Let now $|v|=l+1$ and assume the result holds for all $v^{\prime} \in V^{l}$. We will show that $(x, v)$ is equivalent under $R$ to $\left(f^{*}(x, u), \varepsilon\right)$. Consider $(x, v[l])$. Since $v[l] \in V^{l}$ we know that $\left((x, v[l]),\left(f \ngtr\left(x, u^{\prime}\right), \varepsilon\right)\right) \in R$ for any $u^{\prime} \in U^{l}$ such that $\alpha_{2}^{*}\left(x, u^{\prime}\right)=v[l]$. We also know that $S$ is invariant under $h$ so that:

Since $|v(l+1)|=1$ it follows from (4.5) that:

$$
\left((x, v[l] v(l+1)),\left(f^{*}\left(x, u^{\prime}\right), v(l+1)\right)\right) \in R
$$

$$
\left(\left(f^{*}\left(x, u^{\prime}\right), v(l+1)\right),\left(f^{*}\left(x, u^{\prime} u^{\prime \prime}\right), \varepsilon\right)\right) \in R
$$

for any $u^{\prime \prime} \in U$ such that $\alpha_{2}\left(f^{*}(x, u), u^{\prime \prime}\right)=v(l+1)$ Transitivity of $R$ applied to (4.8) and (4.9) now implies that $(x, v)=(x, v[l] v(l+1))$ is equivalent under $R$ to $\left(f^{*}(x, u), \varepsilon\right)$ where $u=u^{\prime} u^{\prime \prime} \in U^{l+1}$ is any element satisfying $\alpha_{2}^{*}(x, u)=v$.

4.2. Existence of input induced symbolie bisimulations. In this section we characterize which $\alpha_{2}{ }^{-}$ completions result in control systems with finite state set. The finiteness results developed in this section will require the notion of reachable control system.

Definition 4.6. A control system $M \times U \stackrel{f}{\longrightarrow} M$ is said to be reachable from $x_{0} \in M$ if $f_{x_{0}}^{*}: U^{*} \rightarrow M$ is surjective.

We will restrict our attention to maps $\alpha_{2}: M \times U \rightarrow V$ that are fiber surjective onto a finite set $V$. Fiber surjectivity will imply bisimilarity while finiteness of $V$ can be assumed without loss of generality since if $N \times V \stackrel{g}{\longrightarrow} N$ is a control system with finite state set, then we need an input set $V$ with no more than $|N|$ elements as there are no more than $|N|$ states that can be reached from any state in $N$.

Theorem 4.7. Let $M \times U \stackrel{f}{\longrightarrow} M$ be a control system reachable from $x_{0} \in M$ and let $\alpha_{2}: M \times U \rightarrow V$ be a fiber surjective map onto a finite set $V$. The $\alpha_{2}$-completion of $f$ has finite state set iff there exists $k \in \mathbb{N}$ such that $\alpha_{1} \circ f_{\bar{x}_{0}}^{\leq k}: U \leq k \rightarrow N$ is surjective.

Proof. Consider the family of sets $\left\{E_{v}\right\}_{v \in V \leq k}$ where each set $E_{v}$ is defined by the points $x \in M$ for which the following conditions are satisfied:

(1) there exists a $u \in U^{\leq k}$ such that $f \leq k\left(x_{0}, u\right)=x$;

(2) $\alpha_{2}^{*}\left(x_{0}, u\right)=v$.

We first note that by Proposition 4.5 each set $E_{v}$ is totally contained in an equivalence class of $S$. We now claim that $S$ has no more than $\sum_{i=1}^{k}|V|^{i}$ equivalence classes. This follows from the surjectivity assumption 
on $\alpha_{1} \circ f_{\bar{x}_{0}}^{\leq k}: U \leq k \rightarrow N$ which implies that cardinality of $N$ equals the cardinality of the image of $\alpha_{1} \circ f_{\overline{x_{0}}}^{\leq k}$ which, in view of $\alpha_{1} \circ f_{x_{0}}^{\leq k}=g_{\alpha_{1}\left(x_{0}\right)}^{\leq k} \circ \alpha_{2}\left(x_{0}, \cdot\right)$, is bounded by $\sum_{i=1}^{k}|V|^{i}$.

Assume now that the $\alpha_{2}$-completion $g$ of $f$ has finite state set, let $y_{0}=\alpha_{1}\left(x_{0}\right)$ and let $y \in g_{y_{0}}^{n}\left(V^{n}\right)$ for any $n \in \mathbb{N}$. Fiber surjectivity of $\alpha_{2}$ implies fiber surjectivity of $\alpha_{2}^{n}$ from which we conclude existence of $u \in U^{n}$ satisfying $\alpha_{2}^{n}\left(x_{0}, u\right)=v$ for any $v \in V^{n}$ satisfying $g_{y_{0}}^{n}(v)=y$. We now use the fact that $\alpha: f \rightarrow g$ is a morphism to conclude $\alpha_{1} \circ f^{n}\left(x_{0}, u\right)=g^{n}\left(y_{0}, v\right)=g^{n}\left(\alpha_{1}\left(x_{0}\right), \alpha_{2}\left(x_{0}, u\right)\right)$ which shows that $\alpha_{1} \circ f_{x_{0}}^{n}\left(U^{n}\right)=g_{y_{0}}^{n}\left(V^{n}\right)$. To conclude the proof it suffices to show that $g_{y_{0}}^{n}\left(V^{n}\right)=g_{y_{0}}^{\leq k}\left(V^{\leq k}\right)$ for some fixed $k \in \mathbb{N}$. But this follows at once from the observation that finiteness of the state set of $g$ implies that $y$ can be reached from $y_{0}$ in no more than $k=|N|-1$ steps.

Even though Theorem 4.7 provides us with a necessary and sufficient condition for finiteness of input completions, we cannot test surjectivity of $\alpha_{1} \circ f_{x_{0}}^{\leq k}$ without determining $\alpha_{1}$. The following corollary strengths surjectivity of $\alpha_{1} \circ f_{x_{0}}^{\leq k}$ to a condition not requiring a priori knowledge of $\alpha_{1}$.

Corollary 4.8. Let $M \times U \stackrel{f}{\longrightarrow} M$ be a control system reachable from $x_{0} \in M$ and let $\alpha_{2}: M \times U \rightarrow V$ be a fiber surjective map onto a finite set $V$. If there exists $k \in \mathbb{N}$ such that $f_{\overline{x_{0}}}^{\leq k}: U \leq k \rightarrow M$ is surjective then the $\alpha_{2}$-completion of $f$ has finite state set

We can use Corollary 4.8 to identify concrete classes of control systems admitting finite bisimulations.

Corollary 4.9. Let $M \times U \stackrel{f}{\longrightarrow} M$ be a control system satisfying any of the following properties:

(1) $f$ is induced by a discrete-time controllable linear system, that is, $f(x, u)=A x+B u$ with $x \in \mathbb{R}^{n}$, $u \in \mathbb{R}^{m}, A$ and $B$ matrices of appropriate dimensions and the matrix $\left[A^{n-1} B\left|A^{n-2} B\right| \ldots \mid B\right]$ has rank $n$;

(2) $f$ is induced by a continuous-time controllable linear system, that is, $f(x, \mathbf{u})=\mathbf{x}(\tau)$ where $\tau>0, \mathbf{x}$ is the solution of the differential equation $\frac{d}{d t} \mathbf{x}=A \mathbf{x}+B \mathbf{u}$ with $\mathbf{x}(0)=x \in \mathbb{R}^{n}, \mathbf{u}:[0, \tau] \rightarrow \mathbb{R}^{m}$ is an element of the set $U$ of all functions for which $\mathbf{x}$ is uniquely defined on the interval $[0, \tau], A$ and $B$ are matrices of appropriate dimensions, and the matrix $\left[A^{n-1} B\left|A^{n-2} B\right| \ldots \mid B\right]$ has rank $n$;

(3) $f$ is induced by a controllable nonlinear system generating a constant dimensional Lie algebra on a compact manifold, that is, $f(x, \mathbf{u})=\mathbf{x}(\tau)$ where $\tau>0, \mathbf{x}$ is the solution of the differential equation $\frac{d}{d t} \mathbf{x}=F(\mathbf{x}, \mathbf{u})$ with $F: M \times \mathbb{R}^{m} \longrightarrow T M$ a smooth map such that the smallest Lie algebra containing the vector fields $\{F(x, u)\}_{u \in \mathbb{R}^{m}}$ has the same rank at every $x \in M, M$ is a compact smooth manifold, $\mathbf{u}:[0, \tau] \rightarrow \mathbb{R}^{m}$ is an element of the set $U$ of all functions for which $\mathbf{x}$ is uniquely defined on the interval $[0, \tau]$ and $f$ is reachable for every $x \in M$.

then, for any fiber surjective map $\alpha_{2}: M \times U \rightarrow V$ onto a finite set $V$, the $\alpha_{2}$-completion of $f$ has finite state set.

Proof. All the three cases follow from Corollary 4.8 once we establish the existence of $x_{0} \in M$ and $k \in \mathbb{N}$ making $f_{x_{0}}: U^{\leq k} \rightarrow \mathbb{R}^{n}$ surjective.

Standard results in linear systems theory [Son98, AM97] guarantee that under the assumptions listed in (1) the map $f_{x_{0}}^{\leq k}: U^{\leq k} \rightarrow \mathbb{R}^{n}$ is surjective for any $x_{0} \in \mathbb{R}^{n}$ and $k=n$.

Standard results in linear systems theory [Son98, AM97] guarantee that we can find a $\tau>0$ such that by restricting attention to constant input trajectories defined on intervals of duration $\tau>0$ we obtain a discretetime linear control system satisfying all the assumptions of (1).

Regarding (3) we only provide a sketch of the proof since it involves mathematical tools very different from those used in this paper. It follows from standard results in geometric control theory [Jur97] that under the assumptions of (3) if $f^{*}\left(x_{1}, u\right)=x_{2}$ with $u \in U^{l}$ then there is an open set $\mathcal{O}\left(x_{2}\right)$ containing $x_{2}$ such that for every $x_{2}^{\prime} \in \mathcal{O}\left(x_{2}\right)$ there exists a $u^{\prime} \in U^{l}$ satisfying $f\left(x_{1}, u^{\prime}\right)=x_{2}^{\prime}$. Fix now a point $x \in M$, let $\mathcal{C}=\left\{\mathcal{O}\left(x^{\prime}\right)\right\}_{x^{\prime} \in M}$ 
and note that $\mathcal{C}$ is an open cover of $M$. Since $M$ is compact there exists a finite subcover $\mathcal{C}^{\prime}$ of $\mathcal{C}$. If we now define $\eta$ to be maximum of the length of the inputs taking $x$ to points in the (finitely many) open sets in $\mathcal{C}^{\prime}$, we see that $f_{x}^{\leq k}$ is surjective for $k=\eta$.

We now illustrate Corollary 4.9 in the simplest discrete-time controllable linear system:

$$
\begin{aligned}
& x_{1}(t+\tau)=x_{2}(t) \\
& x_{2}(t+\tau)=u(t)
\end{aligned}
$$

where $x=\left(x_{1}, x_{2}\right) \in \mathbb{R}^{2}$ is the state, $u \in \mathbb{R}$ is the input and $\tau>0$. Let $\alpha_{2}$ be defined as in (4.1):

$$
\alpha_{2}(x, u)=\left\{\begin{aligned}
-1 & \text { if } u \leq-1 \\
0 & \text { if }-1<u<1 \\
1 & \text { if } u \geq 1
\end{aligned}\right.
$$

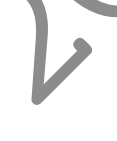

For simplicity we will identify $-1,0,1$ with the symbols $-, 0,+$, respectively and we introduce the sets $U_{-}=$ $\left\{u \in \mathbb{R} \mid \alpha_{2}(u, x)=-1\right\}, U_{0}=\left\{u \in \mathbb{R} \mid \alpha_{2}(u, x)=0\right\}$ and $U_{+}=\left\{u \in \mathbb{R} \mid \alpha_{2}(u, x)=1\right\}$. Starting at the origin and applying the inputs in the symbolic set $V=\{-, 0,+\}$ we construct the following sets:

$$
S_{j}=\left\{\left(x_{1}, x_{2}\right) \in \mathbb{R}^{2} \mid x_{1}=0 \wedge x_{2} \in U_{j}\right\}, \geqslant j \in\{-, 0,+\}
$$

representing points that are identified under equivalence relation $S$ defined in Proposition 4.5. Applying inputs again we obtain a complete description of $S$ given by the following sets defining its equivalence classes:

$$
S_{j}^{i}=\left\{\left(x_{1}, x_{2}\right) \in \mathbb{R}^{2} \mid x_{1} \in U_{i} \wedge x_{2} \in U_{j}\right\}, \quad i, j \in\{-, 0,+\}
$$

The resulting control system $N \times V \stackrel{g}{\longrightarrow} N$ is defined by:

$$
g\left(S_{j}^{i},-\right)=S_{-}^{j} \quad g\left(S_{j, 0}^{i}, 0 S_{0}^{j} \quad g\left(S_{j}^{i},+\right)=S_{+}^{j}, \quad i, j \in\{-, 0,+\}\right.
$$

where $N$ is identified with $\cup_{i, j \in\{-, 0,+\}}\left\{S_{j}^{i}\right\}$ which has 9 elements.

\section{Output inducEd Bisimulations}

A different kind of completion can be obtained by specifying a map redefining the output rather than redefining the input. This motivates the study of symbolic models induced by outputs. We can use again the block example to illustrate the need for this kind of construction. Recall that we are interested in developing a controller to be implemented in embedded hardware/software in order to force the block to a non-zero displacement. Since we do not know the initial displacement of the block, this controller will change the applied force $u$ based on measurements of the block displacement. However, we only have a sensor providing displacement measurements and furthermore the controller implementation can only deal with finitely many values. We can thus assume, for example, that the state $(v, d)$ is transformed into symbols to be used by the controller software/hardware according to the following rule:

$$
\alpha_{3}(v, d)=\left\{\begin{aligned}
-1 & \text { if } d \leq-1 \\
0 & \text { if }-1<d<1 \\
1 & \text { if } d \geq 1
\end{aligned}\right.
$$

It is therefore natural to ask for the existence of an equivalent but simpler model of the continuous dynamics describing the evolution of the symbols $-1,0$ and 1 defining the new output set $Q$. 
5.1. Existence of output induced bisimulations. In this section we consider output completions induced by a map $\alpha_{3}: P \rightarrow Q$ and defined as follows:

Definition 5.1. Let $M \times U \stackrel{f}{\longrightarrow} M \stackrel{r}{\longrightarrow} P$ be a control system and let $\alpha_{3}: P \rightarrow Q$ be a surjective map. The $\alpha_{3}$-completion of $(f, r)$ is a triple $\left((g, s), \alpha_{1}, \alpha_{2}\right)$ where $N \times V \stackrel{g}{\longrightarrow} N \stackrel{s}{\longrightarrow} Q$ is a control system and $\alpha_{1}: M \rightarrow N$ and $\alpha_{2}: M \times U \rightarrow V$ are maps making $\alpha=\left(\alpha_{1}, \alpha_{2}, \alpha_{3}\right): M \times U \times P \rightarrow N \times V \times Q$ a surjective morphism from $(f, r)$ to $(g, s)$ with the following property:

For any control system $N^{\prime} \times V^{\prime} \stackrel{g^{\prime}}{\longrightarrow} N^{\prime} \stackrel{s^{\prime}}{\longrightarrow} Q$ and surjective morphism $\alpha^{\prime}=\left(\alpha_{1}^{\prime}, \alpha_{2}^{\prime}, \alpha_{3}\right):(f, r) \rightarrow\left(g^{\prime}, s^{\prime}\right)$ there exists a unique surjective morphism $\beta=\left(\beta_{1}, \beta_{2}, 1_{Q}\right):\left(g^{\prime}, s^{\prime}\right) \rightarrow(g, s)$ satisfying $\alpha=\beta \circ \alpha^{\prime}$.

Note that although the definition of $\alpha_{3}$-completion is very similar to the definition of $\alpha_{2}$-completion, there is an important difference between the two: the direction of morphism $\beta$. Requiring existence of a morphism $\beta:(g, s) \rightarrow\left(g^{\prime}, s^{\prime}\right)$ would lead to the trivial solution $(g, s)=(f, r)$ and $\alpha=1_{(f, r)}$. However, this reversal in the direction of morphism $\beta$ will require additional assumptions to guarantee existence of $\alpha_{3}$-completions. Part of these assumptions have already been built in the definition of $\alpha_{3}$-completion through the use of the adjective surjective. In particular, requiring $\alpha$ to be surjective guarantees that $\alpha_{3}$-completions are bisimilar to $(f, r)$. The remaining assumptions require the following notion of indistinguishability.

Definition 5.2. Let $M \times U \stackrel{f}{\longrightarrow} M \stackrel{r}{\longrightarrow} P$ be a control system and let $\alpha_{3}: P \rightarrow Q$ be a map. The indistinguishability relations $\left\{\left[\left[\alpha_{3}\right]\right]_{i}\right\}_{i \in \mathbb{N}}$ induced by $\alpha_{3}$ are recursively defined by:

$$
\begin{aligned}
{\left[\left[\alpha_{3}\right]\right]_{0}=\left\{\left(x_{1}, x_{2}\right) \in M \times M \mid \alpha_{3} \circ r\left(x_{1}\right)=\alpha_{3} \circ r\left(x_{2}\right)\right\} } \\
{\left[\left[\alpha_{3}\right]\right]_{i+1}=\left\{\left(x_{1}, x_{2}\right) \in\left[\left[\alpha_{3}\right]\right]_{0} \mid \forall u_{1} \in U \forall u_{2} \in U \wedge \forall u_{2} \in U \exists u_{1} \in U\right.} \\
\left.\left(f\left(x_{1}, u_{1}\right), f\left(x_{2}, u_{2}\right)\right) \in\left[\left[\alpha_{3}\right]\right]_{i}\right\}
\end{aligned}
$$

Two states $x_{1}, x_{2} \in M$ are said to be distinguishable by $\alpha_{3}$ in $n \in \mathbb{N}$ steps if $\left(x_{1}, x_{2}\right) \notin\left[\left[\alpha_{3}\right]\right]_{n}$ and they are said to be distinguishable if there exists $n \in \mathbb{N}$ such that they are distinguishable in $n$ steps. Two states that are not distinguishable are said to be indistinguishable.

The indistinguishability relations introduced in Definition 5.2 are no more than a variation of the sequence of relations leading to the coarsest relation compatible with the transition relation defined by $f$ [Fer90, PT87]. The only difference is the fact that we allow the pairs $\left(f\left(x_{1}, u_{1}\right), f\left(x_{2}, u_{2}\right)\right) \in\left[\left[\alpha_{3}\right]\right]_{i}$ to have different inputs $u_{1}$ and $u_{2}$. This difference is a natural consequence of Definition 3.1 requiring matching of the outputs instead of matching of the labels.

Note that it follows from the definition of indistinguishability relation that if there exists a $k \in \mathbb{N}$ such that $\left[\left[\alpha_{3}\right]\right]_{k}=\left[\left[\alpha_{3}\right]\right]_{k+1}$ then $\left[\left[\alpha_{3}\right]\right]_{k}=\left[\left[\alpha_{3}\right]\right]_{i}$ for all $i \geq k$. In general, the sequence of indistinguishability relations need not stabilize after a finite number of steps. In this case we shall work with the limit relation (see the Appendix for several notions related to relations):

$$
\left[\left[\alpha_{3}\right]\right]=\lim _{i \rightarrow \infty}\left[\left[\alpha_{3}\right]\right]_{i}
$$

which is in facta bisimulation relation between $(f, r)$ and $(f, r)$. Since $\left[\left[\alpha_{3}\right]\right]_{i+1}$ refines $\left[\left[\alpha_{3}\right]\right]_{i}$ and $\pi_{\left[\left[\alpha_{3}\right]\right]_{0}}=$

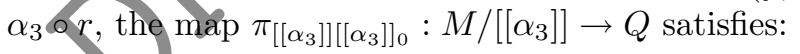

$$
\pi_{\left[\left[\alpha_{3}\right]\right]\left[\left[\alpha_{3}\right]\right]_{0}} \circ \pi_{\left[\left[\alpha_{3}\right]\right]}=\alpha_{3} \circ r
$$

Existence of $\alpha_{3}$-completions can now be characterized by resorting to [[$\left.\left.\alpha_{3}\right]\right]$.

Theorem 5.3. Let $M \times U \stackrel{f}{\longrightarrow} M \stackrel{r}{\longrightarrow} P$ be a control system with a surjective output map and let $\alpha_{3}: P \rightarrow Q$ be a surjective map. If for any $\left(x_{1}, x_{2}\right) \notin\left[\left[\alpha_{3}\right]\right]$ the following equality holds:

$$
\left|\pi_{\left[\left[\alpha_{3}\right]\right]} \circ f\left(x_{1}, U\right)\right|=\left|\pi_{\left[\left[\alpha_{3}\right]\right]} \circ f\left(x_{2}, U\right)\right|
$$

then, the $\alpha_{3}$-completion of $(f, r)$ exists. 
Proof. We construct control system $N \times V \stackrel{g}{\longrightarrow} N$ with $N=M /\left[\left[\alpha_{3}\right]\right]$ and $V$ any set of cardinality $\mid \pi_{\left[\left[\alpha_{3}\right]\right]} \circ$ $f(x, U) \mid$. Set $V$ is independent of the point $x \in M$ since by assumption $\left(x_{1}, x_{2}\right) \notin\left[\left[\alpha_{3}\right]\right]$ implies $\mid \pi_{\left[\left[\alpha_{3}\right]\right]} \circ$ $f\left(x_{1}, U\right)|=| \pi_{\left[\left[\alpha_{3}\right]\right]} \circ f\left(x_{2}, U\right) \mid$ and for $\left(x_{1}, x_{2}\right) \in\left[\left[\alpha_{3}\right]\right]$ it follows from the definition of $\left[\left[\alpha_{3}\right]\right]$ that $\mid \pi_{\left[\left[\alpha_{3}\right]\right]} \circ$ $f\left(x_{1}, U\right)|=| \pi_{\left[\left[\alpha_{3}\right]\right]} \circ f\left(x_{2}, U\right) \mid$. Control system $g$ is defined by letting $g_{y}: V \rightarrow N$ be any isomorphism between $V$ and $\pi_{\left[\left[\alpha_{3}\right]\right]} \circ f(x, U)$ where $x$ is any point satisfying $\pi_{\left[\left[\alpha_{3}\right]\right]}(x)=y$. By definition of $\left[\left[\alpha_{3}\right]\right]$, for any $x_{1}$ and $x_{2}$ such that $\pi_{\left[\left[\alpha_{3}\right]\right]}\left(x_{1}\right)=y=\pi_{\left[\left[\alpha_{3}\right]\right]}\left(x_{2}\right)$ we have $\pi_{\left[\left[\alpha_{3}\right]\right]} \circ f\left(x_{1}, U\right)=\pi_{\left[\left[\alpha_{3}\right]\right]} \circ f\left(x_{2}, U\right)$ thus showing that $g_{y}$ does not depend on the choice of $x$. Furthermore, an isomorphism between $V$ and $\pi_{\left[\left[\alpha_{3}\right]\right]} \circ f(x, U)$ always exists since these sets have the same cardinality. If we now denote $\pi_{\left[\left[\alpha_{3}\right]\right]}$ by $\alpha_{1}$ it follows by construction of $g$ that $\alpha_{1} \circ f(x, U) \subseteq g\left(\alpha_{1}(x), V\right)$ and that $g_{y}$ is injective. Therefore, by Proposition 2.2 there exists a unique map $\alpha_{2}$ making $\left(\alpha_{1}, \alpha_{2}\right)$ a morphism from $f$ to $g$. Note that $\alpha_{2}$ is necessarily fiber surjective since $\alpha_{1} \circ f(x, U)=$ $g\left(\alpha_{1}(x), V\right)$ and $g_{\alpha_{1}(x)}$ is a bijection between $V$ and $g\left(\alpha_{1}(x), V\right)$. In view of (5.2) we equip $g$ with the output map $s=\pi_{\left[\left[\alpha_{3}\right]\right]\left[\left[\alpha_{3}\right]\right]_{0}}$. This results in the desired surjective morphism $\alpha=\left(\alpha_{1}, \alpha_{2}, \alpha_{3}\right):(f, r) \rightarrow(g, s)$ where surjectivity of $s$ follows from surjectivity of $r$ and $\alpha_{3}$.

Let now $N^{\prime} \times V^{\prime} \stackrel{g^{\prime}}{\longrightarrow} N^{\prime} \stackrel{s^{\prime}}{\longrightarrow} Q$ be any other control system equipped with a sưjective morphism $\alpha^{\prime}=$ $\left(\alpha_{1}^{\prime}, \alpha_{2}^{\prime}, \alpha_{3}\right):(f, r) \rightarrow\left(g^{\prime}, s^{\prime}\right)$. Since $\alpha_{2}^{\prime}$ is fiber surjective and $\alpha_{1}$ surjective, the equivalence relation $S \subseteq M \times M$ defined by $\left(x_{1}, x_{2}\right) \in S$ if $\alpha_{1}^{\prime}\left(x_{1}\right)=\alpha_{1}^{\prime}\left(x_{2}\right)$ is a bisimulation relation. Furthermore, $\alpha_{3} \circ r$ factors through $\pi_{S}=\alpha_{1}^{\prime}$ since $\alpha_{3} \circ r=s^{\prime} \circ \alpha_{1}^{\prime}$. However, [[ $\left.\left.\alpha_{3}\right]\right]$ is the coarsest equivalence relation satisfying these properties which implies existence of a surjective map $\pi_{S\left[\left[\alpha_{3}\right]\right]}=\beta_{1}: N^{\prime} \rightarrow N$ satisfying $\beta_{1} \circ \pi_{S}=\pi_{\left[\left[\alpha_{3}\right]\right]}$. We also have:

$$
\begin{array}{rc}
\beta_{1} \circ \pi_{S} \circ f(x, u)=\pi_{\left[\left[\alpha_{3}\right]\right]} \circ f(x, u) & \\
\Rightarrow \beta_{1} \circ g^{\prime}\left(\alpha_{1}^{\prime}(x), \alpha_{2}^{\prime}(x, u)\right)=g\left(\alpha_{1}(x), \alpha_{2}(x, v)\right) & \text { since } \alpha_{1} \text { and } \alpha_{1}^{\prime} \text { are morphisms } \\
\Rightarrow \beta_{1} \circ g^{\prime}\left(\alpha_{1}^{\prime}(x), V^{\prime}\right) \subseteq g\left(\alpha_{1}(x), V\right)=g\left(\beta_{1} \circ \alpha_{1}^{\prime}(x), V\right) & \text { since } S \text { refines }\left[\left[\alpha_{3}\right]\right]
\end{array}
$$

It now follows from injectivity of $g_{y}$ and Proposition 2.2 the existence of a unique map $\beta_{2}$ making $\left(\beta_{1}, \beta_{2}\right)$ a morphism from $g^{\prime}$ to $g$. Fiber surjectivity of $\beta_{2}$ can be shown by the same argument used to show fiber surjectivity of $\alpha_{2}$ in the first part of the proof and it follows from $\beta_{1} \circ \alpha_{1}^{\prime}=\alpha_{1}$ that $\left(\beta_{1}, \beta_{2}\right) \circ\left(\alpha_{1}^{\prime}, \alpha_{2}^{\prime}\right)=\left(\alpha_{1}, \alpha_{2}\right)$. Furthermore, $\left(\beta_{1}, \beta_{2}\right)$ is unique, up to isomorphism, since $\beta_{1}$ is uniquely determined by $\alpha_{1}$ and $\alpha_{1}^{\prime}$ while $\beta_{2}$ is uniquely determined by $\beta_{1}$ and $g$, and $g$ is umique up to the isomorphisms $g_{y}: V \rightarrow N$. To conclude the proof we only need to show that (2.5) is satisfied, that is, that $s^{\prime}=s \circ \beta_{1}$ holds. But this follows from $s \circ \alpha_{1}=\alpha_{3} \circ r=s^{\prime} \circ \alpha_{1}^{\prime}$ combined with: $\beta_{1} \circ \alpha_{1}^{\prime}=\alpha_{1}$ which results in $s \circ \beta_{1} \circ \alpha_{1}^{\prime}=s^{\prime} \circ \alpha_{1}^{\prime}$. Surjectivity of $\alpha_{1}^{\prime}$ allows to simplify the previous expression and to obtain the desired equality $s^{\prime}=s \circ \beta_{1}$.

The assumption expressed by equality (5.3) cannot be dropped since this can prevent the unique factorization property enjoyed by $\alpha_{3}$-completions. This fact is illustrated in the following example. Consider control system $M \times U \stackrel{f}{\longrightarrow} M$ with $M=\left\{x_{1}, x_{2}, x_{3}, x_{3}\right\}, U=\left\{u_{1}, u_{2}\right\}, Q=\left\{q_{1}, q_{2}\right\}$ and defined in Table 3 . In this case we

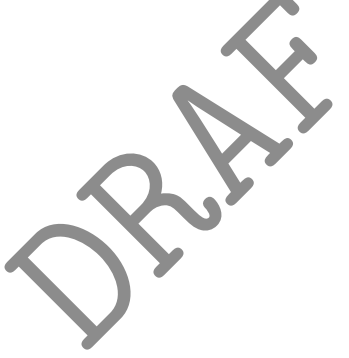

\begin{tabular}{cc|c|c}
$x$ & $u$ & $f(x, u)$ & $\alpha_{3}(x)$ \\
\hline$x_{1}$ & $u_{1}$ & $x_{1}$ & $q_{1}$ \\
$x_{1}$ & $u_{2}$ & $x_{3}$ & $q_{1}$ \\
$x_{2}$ & $u_{1}$ & $x_{2}$ & $q_{1}$ \\
$x_{2}$ & $u_{2}$ & $x_{4}$ & $q_{1}$ \\
$x_{3}$ & $u_{1}$ & $x_{3}$ & $q_{2}$ \\
$x_{3}$ & $u_{2}$ & $x_{4}$ & $q_{2}$ \\
$x_{4}$ & $u_{1}$ & $x_{4}$ & $q_{2}$ \\
$x_{4}$ & $u_{2}$ & $x_{4}$ & $q_{2}$
\end{tabular}

TABLE 3. Definition of control system $f$ and map $\alpha_{3}$.

have $\left[\left[\alpha_{3}\right]\right]_{0}=\left[\left[\alpha_{3}\right]\right]_{1}=\left[\left[\alpha_{3}\right]\right]$. In particular $\pi_{\left[\left[\alpha_{3}\right]\right]}=\alpha_{3}$. Condition (5.3) is violated since:

$$
\left|\alpha_{3} \circ f\left(x_{1}, U\right)\right|=2 \neq 1=\left|\alpha_{3} \circ f\left(x_{3}, U\right)\right|
$$


Furthermore, the cardinality of the input set $V$ of $g$ has to be 2 since $\left|\alpha_{3} \circ f\left(x_{1}, U\right)\right|=2$ and for simplicity we will take $V=U$. We will now show that no control system with state set $N=M /\left[\left[\alpha_{3}\right]\right]$ and input set $U$ satisfies the conclusions of Theorem 5.3. Existence of a morphism from $f$ to $g$, implies that $g$ satisfies:

$$
\alpha_{3} \circ f\left(x_{1}, U\right)=\left\{\left[x_{1}\right],\left[x_{3}\right]\right\} \subseteq g\left(\left[x_{1}\right], V\right), \quad \alpha_{3} \circ f\left(x_{3}, U\right)=\left\{\left[x_{3}\right]\right\} \subseteq g\left(\left[x_{3}\right], V\right)
$$

At state $\left[x_{1}\right], g$ is uniquely determined (up to isomorphism) by the fact that $\alpha_{3} \circ f\left(x_{1}, u_{1}\right)=\alpha_{3} \circ f\left(x_{2}, u_{1}\right)$ and $\alpha_{3} \circ f\left(x_{1}, u_{2}\right)=\alpha_{3} \circ f\left(x_{2}, u_{2}\right)$. We can thus define $g\left(\left[x_{1}\right], u_{1}\right)=\left[x_{1}\right]=\alpha_{3} \circ f\left(x_{1}, u_{1}\right)=\alpha_{3} \circ f\left(x_{2}, u_{1}\right)$ and $g\left(\left[x_{1}\right], u_{2}\right)=\left[x_{3}\right]=\alpha_{3} \circ f\left(x_{1}, u_{2}\right)=\alpha_{3} \circ f\left(x_{2}, u_{2}\right)$. At state $\left[x_{3}\right]$ we must have $g\left(\left[x_{3}\right], u_{1}\right)=g\left(\left[x_{3}\right], u_{2}\right)=\left[x_{3}\right]$. We will now show that there exists no fiber surjective map $\alpha_{2}: M \times U \rightarrow V$ making $\alpha=\left(\alpha_{1}, \alpha_{2}\right): f \rightarrow g$ a morphism such that for any other morphism $\alpha^{\prime}: f \rightarrow g^{\prime}$ there exists a unique morphism $\beta: g^{\prime} \rightarrow g$ satisfying $\beta \circ \alpha^{\prime}=\alpha$. For any fiber surjective map $\alpha_{2}$ we must have $\alpha_{2}\left(x_{3}, u_{1}\right) \neq \alpha_{2}\left(x_{3}, u_{2}\right)$. Let us assume that $\alpha_{2}\left(x_{3}, u_{1}\right)=u_{1}$ and $\alpha_{2}\left(x_{3}, u_{2}\right)=u_{2}$ since the same argument works for $\alpha_{2}\left(x_{3}, u_{1}\right)=u_{2}$ and $\alpha_{2}\left(x_{3}, u_{2}\right)=u_{1}$. Consider now control system $g^{\prime}=g$ and map $\alpha_{2}^{\prime}: M \times U \rightarrow V$ defined by:

$$
\alpha_{2}^{\prime}\left(x_{3}, u_{1}\right)=u_{1}, \quad \alpha_{2}^{\prime}\left(x_{3}, u_{2}\right)=u_{2}, \quad \alpha_{2}^{\prime}\left(x_{4}, u_{1}\right)=\alpha_{2}\left(x_{4}, u_{2}\right), \quad \alpha_{2}^{\prime}\left(x_{4}, u_{2}\right)=\alpha_{2}\left(x_{4}, u_{1}\right)
$$

and inducing morphism $\alpha^{\prime}=\left(\alpha_{1}, \alpha_{2}^{\prime}\right): f \rightarrow g^{\prime}$. Existence of a morphism $\beta: g^{\prime} \rightarrow g$ satisfying $\beta \circ \alpha^{\prime}=\alpha$ implies that:

$$
\begin{aligned}
& \qquad \begin{aligned}
\beta_{2}\left(\left[x_{3}\right], a\right) & =\beta_{2} \circ\left(\alpha_{1}, \alpha_{2}^{\prime}\right)\left(x_{3}, a\right)=\alpha_{2}\left(x_{3}, a\right)=a \\
\beta_{2}\left(\left[x_{3}\right], u_{2}\right) & =\beta_{2} \circ\left(\alpha_{1}, \alpha_{2}^{\prime}\right)\left(x_{3}, u_{2}\right)=\alpha_{2}\left(x_{3}, u_{2}\right)=u_{2}
\end{aligned} \\
& \begin{aligned}
\beta_{2}\left(\left[x_{4}\right], \alpha_{2}^{\prime}\left(x_{4}, a\right)\right)=\beta_{2}\left(\left[x_{3}\right], \alpha_{2}^{\prime}\left(x_{4}, a\right)\right) & \text { since } \alpha_{3}\left(x_{4}\right)=\alpha_{3}\left(x_{3}\right) \\
=\beta_{2}\left(\left[x_{3}\right], \alpha_{2}\left(x_{4}, u_{2}\right)\right) & \text { by definition of } \alpha_{2}^{\prime} \\
=\alpha_{2}\left(x_{4}, u_{2}\right) & \text { since by (5.4) and }(5.5), \beta_{2\left[x_{3}\right]}=1_{U}
\end{aligned}
\end{aligned}
$$

However, at $x_{4}$ we have:

We thus see that $\beta_{2}\left(\left[x_{4}\right], \alpha_{2}^{\prime}\left(x_{4}, a\right)\right)=\alpha_{2}\left(x_{4}, u_{2}\right) \neq \alpha_{2}\left(x_{4}, a\right)$ thus violating the desired equality $\beta \circ \alpha^{\prime}=\alpha$. Therefore, no fiber surjective morphism $\beta: g^{\prime} \rightarrow g$ satisfying $\beta \circ \alpha^{\prime}=\alpha$ can exist. Furthermore, the unique factorization property of $\alpha_{3}$-completions is violated even if we try to define $g$ on a larger set $N$ since in this case there would be several choices of maps $\beta_{1}: M /\left[\left[\alpha_{3}\right]\right] \rightarrow N$ that could be completed to a morphism from $g^{\prime}$ to $g$.

5.2. Existence of output induced symbolic $\alpha_{2}$-completions. We now turn our attention to existence of symbolic $\alpha_{3}$-completions. Since $\left[\left[\alpha_{3}\right]\right]$ is the coarsest bisimulation relation between $(f, r)$ and $(f, r)$ satisfying (5.2) the following corollary of Theorem 5.3 is immediate:

Corollary 5.4. Let $M \times U \stackrel{f}{\longrightarrow} M \stackrel{r}{\longrightarrow} P$ be a control system with surjective output map and let $\alpha_{3}: P \rightarrow Q$ be a surjective map onto a finite set $Q$ for which the $\alpha_{3}$-completion $(g, s)$ of $(f, r)$ exists. Control system $(g, s)$ has finite state set iff there exists a $k \in \mathbb{N}$ such that any pair of distinguishable states by $\alpha_{3}$ is distinguishable in $k$ steps.

Corollary 5.4, by equating finiteness of $\alpha_{3}$-completions to finite distinguishability, suggest that we should identify the class of control systems generating the same outputs after a certain finite number of steps since this guarantees stabilization of the indistinguishability relations after a finite number of steps. We formalize this notion in the next result.

Corollary 5.5. Let $M \times U \stackrel{f}{\longrightarrow} M \stackrel{r}{\longrightarrow} P$ be a control system with surjective output map and let $\alpha_{3}: P \rightarrow Q$ be a surjective map onto a finite set $Q$. If $\left[\left[\alpha_{3}\right]\right]$ satisfies (5.3) and for any $x_{1}, x_{2} \in M$ and $u \in U^{*}$ the following holds:

$$
r \circ f^{*}\left(x_{1}, u[i]\right)=r \circ f^{*}\left(x_{2}, u[i]\right) \quad \forall i \geq k
$$

then the $\alpha_{3}$-completion of $(f, r)$ exists and has finite state set.

We can use Corollary 5.5 to identify concrete classes of control systems admitting finite bisimulations. 
Corollary 5.6. Let $M \times U \stackrel{f}{\longrightarrow} M \stackrel{r}{\longrightarrow} P$ be a control system and let $\alpha_{3}: P \rightarrow Q$ be a surjective map satisfying any of the following assumptions:

(1) $f$ is a discrete-time controllable linear system, that is, $f(x, u)=A x+B u$ with $x \in \mathbb{R}^{n}, u \in \mathbb{R}^{m}, A$ and $B$ are matrices of appropriate dimensions and the matrix $\left[A^{n-1} B\left|A^{n-2} B\right| \ldots \mid B\right]$ has rank $n$;

(2) $f$ is a discrete-time linear system, $\alpha_{3}$ is a linear map and $\left(f, \alpha_{3}\right)$ is output-controllable, that is, $f(x, u)=A x+B u$ with $x \in \mathbb{R}^{n}, u \in \mathbb{R}^{m}, A$ and $B$ matrices of appropriate dimensions, $\alpha_{3}(x)=$ $C x \in \mathbb{R}^{o}$ with $C$ a matrix of appropriate dimensions, and the matrix $\left[C A^{n-1} B\left|C A^{n-2} B \ldots\right| C B\right]$ has rank 0 .

If $\left[\left[\alpha_{3}\right]\right]$ satisfies (5.3) then the $\alpha_{3}$-completion of $f$ exists and has finite state set.

Note that even if $\left[\left[\alpha_{3}\right]\right]$ fails to satisfy (5.3) a finite state bisimilar control system still exists and this is sufficient for most of the applications.

Proof. Let us first assume that $f(x, u)=A x+B u$ is in Brunovsky normal form. For $m=1$ this normal form is given by:

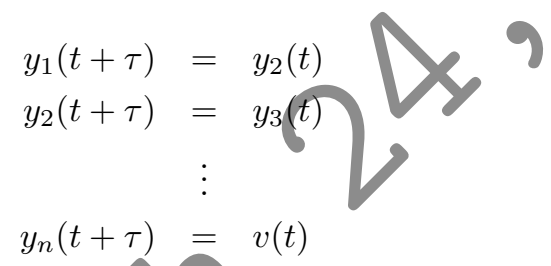

By inspecting (5.6) we immediately conclude that $f^{j+h}(y, v)=(v(j), v(j+1), \ldots, v(j+n))$ for $j \geq 0$ which implies $r \circ f^{*}(y, v[i])=r \circ f^{*}\left(y^{\prime}, v[i]\right)$ for $i \geq n$ and for any map $r: \mathbb{R}^{n} \rightarrow P$. The assumptions of Corollary 5.5 are thus satisfied. For $m>1$ a similar argument applies since in this case the Brunovsky normal form is given by several blocks of the form (5.6). For general controllable linear systems the result follows from the previous argument and from the fact [Son98, AM97] that any controllable linear system is isomorphic to a linear system in Brunovsky normal form.

By Kalman's decomposition Theorem [Son98, AM97] any linear control system can be decomposed as:

$$
\left[\begin{array}{l}
x_{1}(t+1) \\
x_{2}(t+1)
\end{array}\right]=\left[\begin{array}{cc}
A_{11} & A_{12} \\
\mathbf{0} & A_{22}
\end{array}\right]\left[\begin{array}{l}
x_{1}(t) \\
x_{2}(t)
\end{array}\right]+\left[\begin{array}{c}
B_{1} \\
\mathbf{0}
\end{array}\right] u(t)
$$

where $x_{1}(t+1)=A_{1} x_{1}(t)+A_{12} x_{2}(t)+B_{1} u(t)$ is controllable. It then follows from the previous argument that any linear control system equipped with an output map $r: M=\mathbb{R}^{n} \rightarrow P$ admits a finite bisimulation provided that $r$ factors as $r=s \propto \pi$ for any map $s: \mathbb{R}^{o} \rightarrow P$ and for the projection $\pi(x)=x_{1}$. This factorization condition can be checked by determining if the matrix $\left[C B|C A C| \ldots \mid C A^{n-1} B\right]$ defines a surjective linear transformation when $r$ is a linear transformation and $C$ is its matrix representation.

Let as consider again the simplest discrete-time controllable linear system:

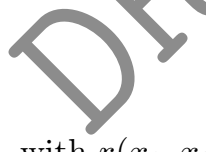

$$
\begin{aligned}
& x_{1}(t+\tau)=x_{2}(t) \\
& x_{2}(t+\tau)=u(t)
\end{aligned}
$$

with $r\left(x_{1}, x_{2}\right)=\left(x_{1}, x_{2}\right)$ and let $\alpha_{3}$ be given by:

$$
\alpha_{3}(x)=\left\{\begin{aligned}
-1 & \text { if } x_{1} \leq-1 \\
0 & \text { if }-1<x_{1}<1 \\
1 & \text { if } x_{1} \geq 1
\end{aligned}\right.
$$

The relation $\left[\left[\alpha_{3}\right]\right]_{0}$ is defined the following three equivalence classes:

$$
S_{j}=\left\{\left(x_{1}, x_{2}\right) \in \mathbb{R}^{2} \mid x_{1} \in X_{j}\right\}, \quad j \in\{-, 0,+\}
$$


where $\left.\left.\left.X_{-}=\right]-\infty,-1\right], X_{0}=\right]-1,1\left[\right.$ and $X_{+}=\left[1, \infty\left[\right.\right.$. Relation $\left[\left[\alpha_{3}\right]\right]_{1}$ satisfies $\left[\left[\alpha_{3}\right]\right]_{1}=\left[\left[\alpha_{3}\right]\right]_{2}$ and is defined by the equivalence classes:

$$
S_{j}^{i}=\left\{\left(x_{1}, x_{2}\right) \in \mathbb{R}^{2} \mid x_{1} \in X_{i} \wedge x_{2} \in X_{j}\right\}, \quad j \in\{-, 0,+\}
$$

The $\alpha_{3}$-completion is now given by:

$$
g\left(S_{j}^{i}, X_{-}\right)=S_{-}^{j} \quad g\left(S_{j}^{i}, X_{0}\right)=S_{0}^{j} \quad g\left(S_{j}^{i}, X_{+}\right)=S_{+}^{j}, \quad i, j \in\{-, 0,+\}
$$

where $V$ is identified with $\cup_{i \in\{-, 0,+\}}\left\{X_{i}\right\}$ and $N$ identified with $\cup_{i, j \in\{-, 0,+\}}\left\{S_{j}^{i}\right\}$. The fact that the $\alpha_{3}$ completion is the same as the $\alpha_{2}$-completion presented in Section 4 for the same control system is due to the fact that we have chosen a control system of a very special form (Brunovsky normal form). In general, $\alpha_{2}$-completions and $\alpha_{3}$-completions are not isomorphic.

\section{Discussion}

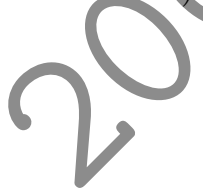

In this paper we have presented two very general constructions on the class of control systems: $\alpha_{2}$-completions and $\alpha_{3}$-completions. The first class of completions is mathematically very natural since no conditions are necessary for its existence. This implies that Theorem 4.3 is valid in a variety of different contexts obtained by imposing different structure on control systems (linearity, smoothness, analyticity, etc). This is in sharp contrast with $\alpha_{3}$-completions which exist under stricter conditions. One of those conditions is surjectivity of the involved morphisms which has to be considerably strengthened when more structure is imposed such as smoothness for example. This is a consequence of Theorem 5.3 being based on the fact that every surjective map $\phi: A \rightarrow B$ can be seen as the projection from $A$ to $A / S$ where $S$ is the equivalence relation on $A$ identifying points $a_{1}$ and $a_{2}$ when $\phi\left(a_{1}\right)=\phi\left(a_{2}\right)$.

A third type of completion could also be considered if one starts with a control system and a map $\alpha_{1}: M \rightarrow N$ between state sets. However, since our objective isoto obtain bisimilar models, either the graph of $\alpha_{1}$ already defines a bisimulation or there is no possibility of obtaining a bisimilar model by constructing a control system with state set $N$. This fact justifies why $\alpha_{1}$-completions have not been considered in this paper.

Even though we make a distinction between inputs and outputs this distinction is not natural in many systems and it would be interesting to extend the results of this paper by dropping this distinction. Such behavioral approach [PW97] could help understand how to relate the sufficient conditions for the existence of symbolic models provided by Corollaires 4.8 and 5.5 and lead to a deeper understanding of which control systems are essentially finite.

On going research is focusing on extending and characterizing new classes of systems satisfying the sufficient conditions for the existence of finite completions. While Corollary 4.8 requires reachability in a finite number of steps, Corollary 5.5 requires a finite output controllability or tracking assumption ensuring that the effect of the initial state will only influence the output in a finite number of steps. Even though the conditions required by Corollary 5.5 appear to be stronger than those required by Corollary 4.8, the possibility of choosing the output map appears to provide greater flexibility in the use of Corollary 5.5.

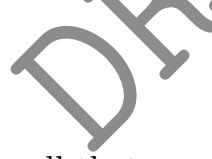

\section{APPENDIX}

Recall that an equivalence relation $R$ on a set $A$ is a subset $R \subseteq A \times A$ satisfying:

(1) Reflexivity: $\forall a \in M,(a, a) \in R$;

(2) Symmetry: $\forall a_{1}, a_{2} \in M,\left(a_{1}, a_{2}\right) \in R$ implies $\left(a_{2}, a_{1}\right) \in R$;

(3) Transitivity: $\left(a_{1}, a_{2}\right) \in R$ and $\left(a_{2}, a_{3}\right) \in R$ implies $\left(a_{1}, a_{3}\right) \in R$.

Given an equivalence relation $R \subseteq A \times A$, we denote by $[a]$ the equivalence class containing $a \in A$ and by $\pi_{R}: A \rightarrow A / R$ the (surjective) natural projection map taking each point $a \in A$ to its equivalence class $[a]$. 
Conversely, any surjective map $\phi: A \rightarrow B$ defines an equivalence relation on $A$ whose equivalence classes are given by $\phi^{-1}(b)$ for each $b \in B$.

An equivalence relation $R_{2} \subseteq A \times A$ is said to refine equivalence relation $R_{1} \subseteq A \times A$ if for every equivalence class $E_{2}$ of $R_{2}$ there is an equivalence class $E_{1}$ of $R_{1}$ such that $E_{2} \subseteq E_{1}$. Equivalently, $R_{2}$ refines $R_{1}$ if there exists a map $\pi_{R_{2} R_{1}}: A / R_{2} \rightarrow A / R_{1}$ satisfying $\pi_{R_{2} R_{1}} \circ \pi_{R_{2}}=\pi_{R_{1}}$. Map $\pi_{R_{2} R_{1}}$ takes an equivalence class $E_{2}$ of $R_{2}$ into the unique equivalence class $\pi_{R_{2} R_{1}}\left(E_{2}\right)$ of $R_{1}$ containing $E_{2}$.

Any arbitrary relation $R \subseteq A \times A$ can be completed to an equivalence relation by adding the identity relation $I=\left\{\left(a_{1}, a_{2}\right) \in A \times A \mid a_{1}=a_{2}\right\}$ to $R$, resulting in $R \cup I$, and by closing $R \cup I$ under reflexivity and transitivity. This closure $\bar{R}$ is called the equivalence relation generated by $R$ and it is the coarsest equivalence relation containing the pairs $\left(a_{1}, a_{2}\right) \in R$ in the sense that any other equivalence relation containing the pairs $\left(a_{1}, a_{2}\right) \in R$ is a refinement of $\bar{R}$.

Consider now an equivalence relation $R \subseteq A \times A$ and a map $f: A \rightarrow A$. Relation $R$ is said to be invariant under map $f$ when $\left(a_{1}, a_{2}\right) \in R$ implies $\left(f\left(a_{1}\right), f\left(a_{2}\right)\right) \in R$. In this case $f$ induces a map $f / R: A / R \rightarrow A / R$ defined by $(f / R)([a])=[f(a)]$. Map $f / R$ is well define since for any $a_{2}, a_{3} \in\left[a_{1}\right]$ we have $\left(f\left(a_{2}\right), f\left(a_{1}\right)\right) \in R$ and $\left(f\left(a_{3}\right), f\left(a_{1}\right)\right) \in R$. Consequently, $\left[f\left(a_{2}\right)\right]=\left[f\left(a_{3}\right)\right]=\left[f\left(a_{1}\right)\right]=(f / R)\left(\left[a_{1}\right]\right)$. If an equivalence relation $R$ is not invariant under a map $f$, we can close it under $f$ resulting in thecoarsest equivalence relation $\bar{R}$ containing the pairs $\left(a_{1}, a_{2}\right) \in R$ and invariant under $f$, that is, any other equivalence relation containing the pairs $\left(a_{1}, a_{2}\right) \in R$ and invariant under $f$ is a refinement of $\bar{R}$.

Consider now a sequence of equivalence relations $\left\{R_{i}\right\}_{i \in \mathbb{N}}$ on a set $A$ where for each $i \in \mathbb{N}, R_{i+1}$ is a refinement of $R_{i}$. The limit of this sequence is an equivalence relation $R=\lim _{i \rightarrow \infty} R_{i}$ refining every equivalence relation $R_{i}$ and such that for any other equivalence relation $S$ refining every equivalence relation $R_{i}, S$ is a refinement of $R$.

$\left[\mathrm{ACH}^{+} 95\right]$ R. Alur, C.Courcoubetis, N. Halbwachs, T.A. Henzinger, P.H. Ho, X. Nicollin, A.Olivero, J. Sifakis, and S. Yovine. Hybrid automata: An algorithmic approach to specification and verification of hybrid systems. Theoretical Computer Science, 138:3-34, 1995.

[AD94] Rajeev Alur and David L. Dill A theory of timed automata. Theoretical Computer Science, 126(2):183-235, 1994.

[AM74] Michael Arbib and Ernest G. Manes. Machines in a category: An expository introduction. SIAM Review, 16(2):163192, 1974.

[AM97] Panos J. Antsaklisand Anthony N. Michel. Linear Systems. McGraw-Hill, 1997.

[ASL93] P. J. Antsaklis, J. A.Stiver, and M. D. Lemmon. Hybrid system modeling and autonomous control systems. In R. L. Grossman, A. Nerode, A. P. Ravn, and H. Rischel, editors, Hybrid Systems, volume 736 of Lecture Notes in Computer Sience,pages 366-392. Springer-Verlag, 1993.

[BMP02] A. Bicchi,A. Marigo, and B. Piccoli. On the rechability of quantized control systems. IEEE Transaction on Automatic Control, April 2002.

[BMRT04] Thomas Brihaye, Christian Michaux, Cédric Riviére, and Christophe Troestler. On o-minimal hybrid systems. In Rajeev Alur and George J. Pappas, editors, Hybrid Systems: Computation and Control, volume 2993 of Lecture

Notes in Computer Science, pages 219-233. Springer-Verlag, 2004 .
Mireille Broucke. A geometric approach to bisimulation and verification of hybrid systems. In Fritz W. Vaandrager
[Bro99] Jan H. van Schuppen, editors, Hybrid Systems: Computation and Control, volume 1569 of Lecture Notes in and Jan H. van Schuppen, editors, Hybrid Systems: Cor
Computer Science, pages 61-75. Springer-Verlag, 1999.

[CW98] P.E. Caines and Y.J. Wei. Hierarchical hybrid control systems: A lattice theoretic formulation. IEEE Transactions on Automatic Control : Special Issue on Hybrid Systems, 43(4):501-508, April 1998.

[DMN02] J. M. Davoren, T. Moor, and A. Nerode. Hybrid control loops, A/D maps and dynamic specifications. In Claire Tomlin and Mark R. Greenstreet, editors, Hybrid Systems: Computation and Control, volume 2289 of Lecture Notes in Computer Sience. Springer-Verlag, 2002.

[Fer90] J.-C. Fernandez. An implementation of an efficient algorithm for bisimulation equivalence. Science of Computer Programming, 13:219-236, 1989/90.

[Gog73] J.A. Goguen. Realization is universal. Mathematical Systems Theory, 6(4):359-374, 1973.

[HKPV98] Thomas A. Henzinger, Peter W. Kopke, Anuj Puri, and Pravin Varaiya. What's decidable about hybrid automata? Journal of Computer and System Sciences, 57:94-124, 1998. 
[HM00] T.A. Henzinger and R. Majumdar. Symbolic model checking for rectangular hybrid systems. In S. Graf, editor, TACAS 2000: Tools and algorithms for the construction and analysis of systems, Lecture Notes in Computer Science, New-York, 2000. Springer-Verlag.

[HSC02] In Claire Tomlin and Mark R. Greenstreet, editors, Hybrid Systems: Computation and Control 2002, volume 2289 of Lecture Notes in Computer Science. Springer-Verlag, Stanford, CA, USA, 2002.

[HSC03] In Oded Maler and Amir Pnueli, editors, Hybrid Systems: Computation and Control 2003, volume 2623 of Lecture Notes in Computer Science. Springer-Verlag, 2003.

[HSC04] In Rajeev Alur and George J. Pappas, editors, Hybrid Systems: Computation and Control 2004, volume 2993 of Lecture Notes in Computer Science. Springer-Verlag, Philadelphia, PA, USA, 2004.

[HSC05] In Manfred Morari and Lothar Thiele, editors, Hybrid Systems: Computation and Control 2005, volume 3414 of Lecture Notes in Computer Science. Springer-Verlag, Zurich, Switzerland, 2005.

[HSC06] In Joao P. Hespanha and Ashish Tiwari, editors, Hybrid Systems: Computation and Control 2006, volume 3927 of Lecture Notes in Computer Science. Springer-Verlag, Santa Barbara, CA, USA, 2006.

[HTP05] Esfandiar Haghverdi, Paulo Tabuada, and George J. Pappas. Bisimulation relations for dynamical, control and hybrid systems. Theoretical Computer Science, 34(2-3):387-392, 2005.

[Jur97] Velimir Jurdjevic. Geometric Control Theory. Number 51 in Cambridge Studies in Advanced Mathematics. Cambridge University Press, 1997.

[KA03] X. Koutsoukos and P. Antsaklis. Safety and reachability of piecewise linear hybrid dynamical systems based on discrete abstractions. Journal of Discrete Event Dynamic Systems: Theory and Applications, 13(3):203-243, 2003.

[Lan71] Saunders Mac Lane. Categories for the Working Mathematician. Springer-Verlag, 1971.

[LPS00] Gerardo Lafferriere, George J. Pappas, and Shankar Sastry. O-minimal hybrid systems. Mathematics of Control, Signals and Systems, 13(1):1-21, March 2000.

[Lun94] J. Lunze. Qualitative modelling of linear dynamical systems with quantized state measurements. Automatica, 30:417431, 1994.

[Mil89] R. Milner. Communication and Concurrency. Prentice Hall, 1989.

[MRO02] T. Moor, J. Raisch, and S.D. O'Young. Discrete supervisory control of hybrid systems based on l-complete approximations. Journal of Discrete Event Dynamical Systems, 12(1):83-107, 2002.

[NK93] Anil Nerode and Wolf Kohn. Models for hybrid systems: Automata, topologies, controllability, observability. In Robert L. Grossman, Anil Nerode, Anders P. Ravn, and Hans Rischel, editors, Hybrid Systems, volume 736 of Lecture Notes in Computer Sience, pages 317-356. Springer-Verlag, 1993.

[Pap03] George J. Pappas. Bisimilar linear systems. Automatica, 39(12):2035-2047, December 2003.

[Par81] D.M.R. Park. Concurrency and automata on infinite sequences. volume 104 of Lecture Notes in Computer Science, pages 167-183, 1981.

[PLPB02] Stefania Pancanti, Laura Leonardi, Lucia Pallottino, and Antonio Bicchi. Optimal control of quantized linear systems. In Claire Tomlin and Mark R. Greenstreet, editors, Hybrid Systems: Computation and Control, Lecture Notes in Computer Sience, pages 351-363. Springer-Verlag, 2002.

[PT87] Robert Paige and Robert Tarjan. Three partition refinement algorithms. SIAM Journal on Computing, 16(6):973989, 1987.

[PV94] A. Puri and P. Varaiya. Decidability of hybrid systems with rectangular inclusions. In Computer Aided Verification, pages 95-104, 1994.

[PvdSdB04] G. Pola, A. J. vander Schaft, and M. D. di Benedetto. Bisimulation theory for switching linear systems. In Proceedings of the 43rd IEEE Conference on Decision and Control, pages 1406-1411, Paradise Island, Bahamas, 2004.

[PW97] J. W. Polderman and J.C. Willems. Introduction to Mathematical Systems Theory: A Behavioral Approach. SpringerVerlag, New York, 1997.

[RO98] J. Raisch and S.D. O'Young. Discrete approximations and supervisory control of continuous systems. IEEE Transactions on Automatic Control : Special Issue on Hybrid Systems, 43(4):569-573, April 1998.

[SKA01] J.A. Stiver, X.D. Koutsoukos, and P.J. Antsaklis. An invariant based approach to the design of hybrid control systems. International Journal of Robust and Nonlinear Control, 11(5):453-478, 2001.

[SKHP97] Olaf Stursberg, Stefan Kowalewski, Ingo Hoffmann, and Jorg Preussig. Comparing timed and hybrid automata as approximations of continuous systems. In Panos J. Antsaklis, Wolf Kohn, Anil Nerode, and Shankar Sastry, editors, Hybrid Systems IV, volume 1273 of Lecture Notes in Computer Sience, pages 361-377. Springer-Verlag, 1997.

[Son98] Eduardo D. Sontag. Mathematical Control Theory, volume 6 of Texts in Applied Mathematics. Springer-Verlag, New-York, 2nd edition, 1998.

[TMD04] D. C. Tarraf, A. Megretski, and M. A. Dahleh. Finite automata approximations with error bounds for systems with quantized actuation and measurement: A case study. In Proceedings of the 43rd IEEE Conference on Decision and Control, pages 1436-1441, Paradise Island, Bahamas, 2004.

[TP03a] Paulo Tabuada and George J. Pappas. Finite bisimulations of controllable linear systems. In Proceedings of the 42nd IEEE Conference on Decision and Control, Hawaii, 2003.

[TP03b] Paulo Tabuada and George J. Pappas. Model checking LTL over controllable linear systems is decidable. In Oded Maler and Amir Pnueli, editors, Hybrid Systems: Computation and Control, volume 2623 of Lecture Notes in Computer Sience. Springer-Verlag, 2003. 
[TP04] P. Tabuada and G. J. Pappas. Bisimilar control affine systems. Systems and Control Letters, 52(1):49-58, 2004.

[TP06] Paulo Tabuada and George J. Pappas. Linear Time Logic control of discrete-time linear systems. IEEE Transaction on Automatic Control, 2006. Accepted for publication, available at http://www.nd.edu/ ptabuada.

[vdS04] A.J. van der Schaft. Equivalence of dynamical systems by bisimulation. IEEE Transactions on Automatic Control, 49(12):2160-2172, 2004.

$\left[\mathrm{VSS}^{+}\right.$01] R. Vidal, S. Schaffert, O. Shakernia, J. Lygeros, and S. Sastry. Decidable and semi-decidable controller synthesis for classes of discrete time hybrid systems. In Proceedings of the 40th IEEE Conference on Decision and Control, pages 1243-1248, Orlando, December 2001.

[WN94] Glynn Winskel and Mogens Nielsen. Models for concurrency. In Abramsky, Gabbay, and Maibaum, editors, Handbook of Logic and Foundations of Theoretical Computer Science, volume 4. Oxford University Press, London, 1994.

Department of Electrical Engineering, 268 Fitzpatrick Hall, University of Notre Dame, Notre Dame, in 46556 , HTTP://WWW.ND.EDU/ PTABUADA

E-mail address: ptabuada@nd.edu
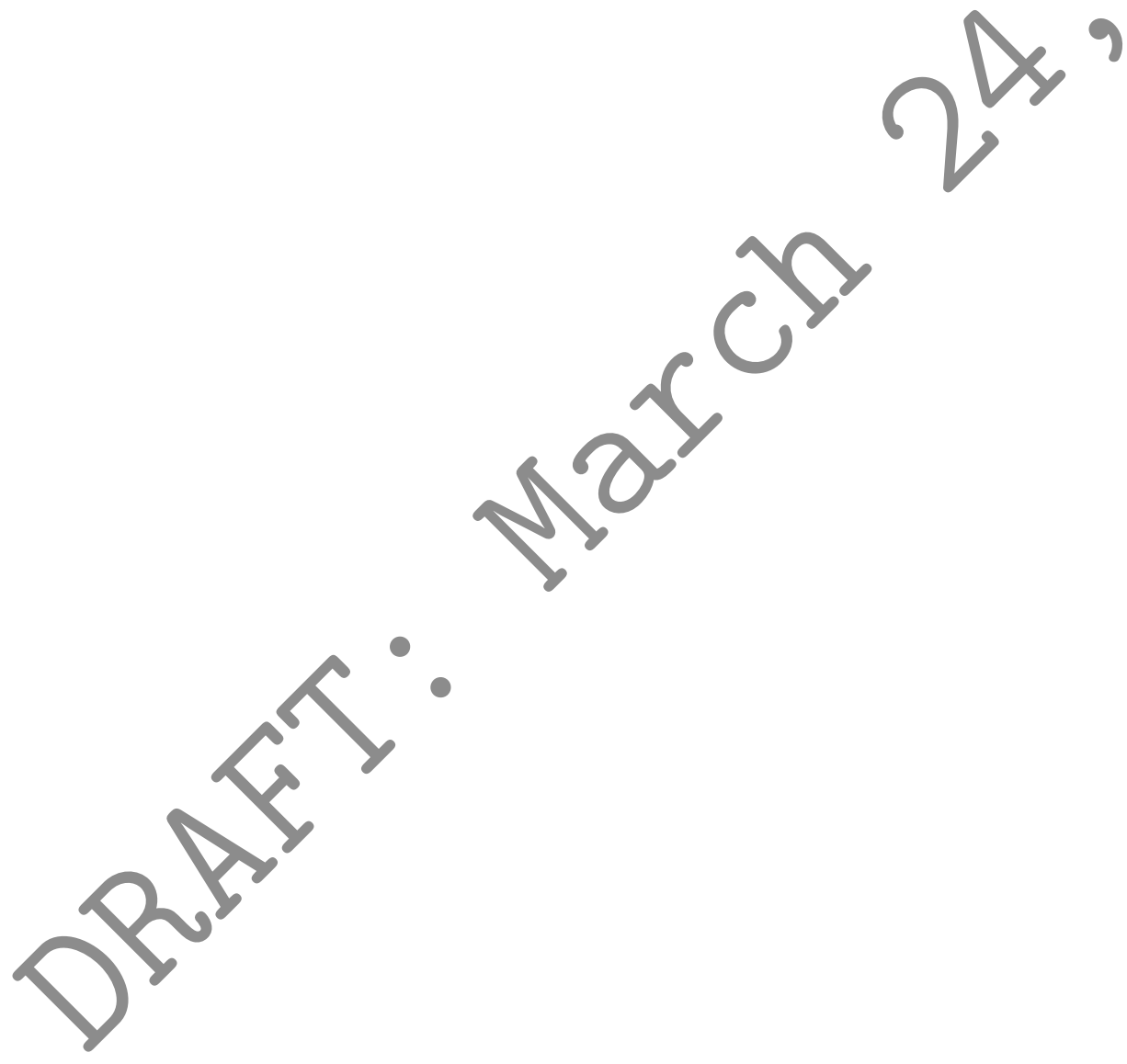